\title{
Multicritical behavior in frustrated spin systems with noncollinear order
}

\author{
Pasquale Calabrese, ${ }^{1}$ Andrea Pelissetto, ${ }^{2}$ and Ettore Vicari ${ }^{3}$ \\ ${ }^{1}$ R. Peierls Center for Theoretical Physics, University of Oxford, \\ 1 Keble Road, Oxford OX1 3NP, United Kingdom. \\ 2 Dip. Fisica dell'Università di Roma "La Sapienza" and INFN, \\ P.le Moro 2, I-00185 Roma, Italy \\ 3 Dip. Fisica dell'Università di Pisa and INFN, V. Buonarroti 2, I-56127 Pisa, Italy \\ e-mail: calabres@df.unipi.it, Andrea.Pelissetto@roma1.infn.it, vicari@df.unipi.it
}

(June 27, 2021)

\begin{abstract}
We investigate the phase diagram and, in particular, the nature of the the multicritical point in three-dimensional frustrated $N$-component spin models with noncollinear order in the presence of an external field, for instance easyaxis stacked triangular antiferromagnets in the presence of a magnetic field along the easy axis. For this purpose we study the renormalization-group flow in a Landau-Ginzburg-Wilson $\phi^{4}$ theory with symmetry $\mathrm{O}(2) \otimes\left[\mathbb{Z}_{2} \oplus \mathrm{O}(N-1)\right]$ that is expected to describe the multicritical behavior. We compute its $\overline{\mathrm{MS}}$ $\beta$ functions to five loops. For $N \geq 4$, their analysis does not support the hypothesis of an effective enlargement of the symmetry at the multicritical point, from $\mathrm{O}(2) \otimes\left[\mathbb{Z}_{2} \oplus \mathrm{O}(N-1)\right]$ to $\mathrm{O}(2) \otimes \mathrm{O}(N)$. For the physically interesting case $N=3$, the analysis does not allow us to exclude the corresponding symmetry enlargement controlled by the $\mathrm{O}(2) \otimes \mathrm{O}(3)$ fixed point. Moreover, it does not provide evidence for any other stable fixed point. Thus, on the basis of our field-theoretical results, the transition at the multicritical point is expected to be either continuous and controlled by the $\mathrm{O}(2) \otimes \mathrm{O}(3)$ fixed point or to be of first order.
\end{abstract}

PACS Numbers: 64.60.Kw, 05.10.Cc, 05.70.Jk 


\section{INTRODUCTION}

Frustrated spin models with noncollinear order, such as easy-axis stacked triangular antiferromagnets (STAs), reveal a quite complex phase diagram in the presence of an external magnetic field $H$ along the easy axis, see, e.g., Refs. [1-9]. A model of these systems is obtained by considering a stacked triangular lattice, three-component spins $s_{i}$ defined at the sites of the lattice satisfying $s_{i} \cdot s_{i}=1$, and the Hamiltonian

$$
\mathcal{H}_{\mathrm{STA}}=\frac{\beta}{2} \sum_{i j} J_{i j} s_{i} \cdot s_{j}+\sum_{i}\left[D s_{i, z}^{2}+H s_{i, z}\right],
$$

with an antiferromagnetic hopping term $J_{i j}$. For small magnetic fields, one observes two critical lines that are expected to belong to the XY universality class according to the theoretical analysis [10-13]. For large magnetic fields, there is instead a single critical line that is expected to belong to the $\mathrm{O}(2) \otimes \mathrm{O}(2)$ universality class, which is characterized by the symmetry-breaking pattern $\mathrm{O}(2) \otimes \mathrm{O}(2) \rightarrow \mathrm{O}(2)_{\text {diag. }}$. Finally, the large- $H$ and small- $H$ domain are separated by a first-order spin-flop line. These four critical lines meet at a tetracritical point, see Fig. 1. The authors of Ref. [12] argued that the critical behavior at the multicritical point belongs to the $\mathrm{O}(2) \otimes \mathrm{O}(3)$ universality class, with symmetry-breaking pattern $\mathrm{O}(2) \otimes \mathrm{O}(3) \rightarrow \mathbb{Z}_{2} \otimes \mathrm{O}(2)_{\text {diag }}$, described by the Hamiltonian [14]

$$
\begin{aligned}
\mathcal{H}_{\mathrm{sym}}= & \int d^{d} x\left\{\frac{1}{2} \sum_{a i}\left[\sum_{\mu}\left(\partial_{\mu} \Phi_{a i}\right)^{2}+r \Phi_{a i}^{2}\right]\right. \\
& \left.+\frac{g_{1,0}}{4 !}\left(\sum_{a i} \Phi_{a i}^{2}\right)^{2}+\frac{g_{2,0}}{4 !}\left[\sum_{i, j}\left(\sum_{a} \Phi_{a i} \Phi_{a j}\right)^{2}-\left(\sum_{a i} \Phi_{a i}^{2}\right)^{2}\right]\right\},
\end{aligned}
$$

where $\Phi_{a i}$ is a $3 \times 2$ matrix, i.e., $a=1,2,3$ and $i=1,2$. The analysis of Ref. [12] is however not complete, since only the quadratic perturbations of the $\mathrm{O}(2) \otimes \mathrm{O}(3)$ fixed point $(\mathrm{FP})$ were considered. In this paper we reconsider the issue, by performing a complete analysis of all quadratic and quartic perturbations induced by the easy-axis anisotropy, including also those terms that are absent in the theoretical analysis of Ref. [12].

The Landau-Ginzburg-Wilson (LGW) Hamiltonian that describes the multicritical behavior is the most general Hamiltonian with symmetry $\mathrm{O}(2) \otimes\left[\mathbb{Z}_{2} \oplus \mathrm{O}(2)\right]$. It is given by

$$
\begin{aligned}
\mathcal{H}= & \int d^{d} x\left\{\frac{1}{2} \sum_{a i}\left[\sum_{\mu}\left(\partial_{\mu} \phi_{a i}\right)^{2}+r_{\phi} \phi_{a i}^{2}\right]+\frac{1}{2} \sum_{i}\left[\sum_{\mu}\left(\partial_{\mu} \psi_{i}\right)^{2}+r_{\psi} \psi_{i}^{2}\right]\right. \\
& +\frac{u_{0}}{4 !}\left(\sum_{a i} \phi_{a i}^{2}+\sum_{i} \psi_{i}^{2}\right)^{2}+\frac{v_{0}}{4 !}\left[\sum_{i, j}\left(\sum_{a} \phi_{a i} \phi_{a j}+\psi_{i} \psi_{j}\right)^{2}-\left(\sum_{a i} \phi_{a i}^{2}+\sum_{i} \psi_{i}^{2}\right)^{2}\right] \\
& \left.+\frac{w_{0}}{4 !}\left(\sum_{a i} \phi_{a i}^{2}\right)^{2}+\frac{y_{0}}{4 !}\left(\sum_{i} \psi_{i}^{2}\right)^{2}+\frac{z_{0}}{4 !} \sum_{i j}\left[\left(\sum_{a} \phi_{a i} \phi_{a j}\right)^{2}-\sum_{a} \phi_{a i}^{2} \sum_{a} \phi_{a j}^{2}\right]\right\},
\end{aligned}
$$

where $\phi_{a i}$ and $\psi_{i}$ are real fields with $a=1,2$ and $i=1,2$. The LGW Hamiltonian (1.3) can also be obtained from model (1.1) by performing a Hubbard-Stratonovitch transformation $[15,16]$ and an expansion in terms of the critical modes. 


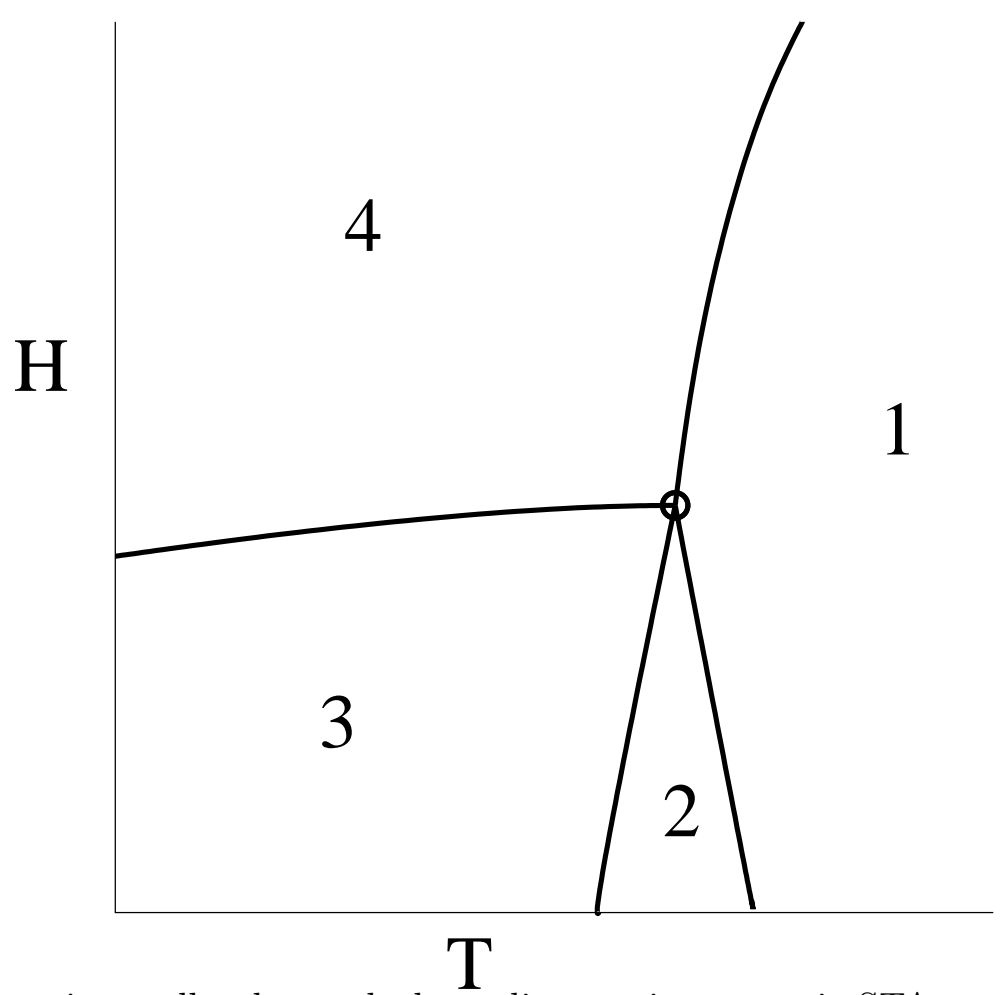

FIG. 1. The experimentally observed phase diagram in easy-axis STAs as a function of temperature $T$ and magnetic field $H$.

The critical behavior at the multicritical point is determined by the stable $\mathrm{FP}$ of the renormalization-group (RG) flow when both $r_{\phi}$ and $r_{\psi}$ are tuned to their critical value. See, e.g., Refs. $[17,18]$ and references therein for a discussion of the field-theoretical (FT) approach to multicritical phenomena. If no stable FP exists or if the system is not in the attraction domain of the stable FP, the transition at the multicritical point is expected to be of first order. The hypothesis of the effective enlargement of the symmetry

$$
\mathrm{O}(2) \otimes\left[\mathbb{Z}_{2} \oplus \mathrm{O}(2)\right] \rightarrow \mathrm{O}(2) \otimes \mathrm{O}(3)
$$

at the multicritical point requires that the $\mathrm{O}(2) \otimes \mathrm{O}(3)$ chiral FP is stable with respect to the quartic terms that break $\mathrm{O}(2) \otimes \mathrm{O}(3)$ to $\mathrm{O}(2) \otimes\left[\mathbb{Z}_{2} \oplus \mathrm{O}(2)\right]$ - those proportional to $w_{0}, y_{0}$, and $z_{0}$ in Hamiltonian (1.3). If this does not occur, the $\mathrm{O}(2) \otimes \mathrm{O}(3) \mathrm{FP}$ does not control the multicritical behavior for generic values of the Hamiltonian parameters. As a consequence, the effective enlargement of the symmetry to $\mathrm{O}(2) \otimes \mathrm{O}(3)$ at the multicritical point requires an additional tuning of the parameters: beside tuning $r_{\phi}$ and $r_{\psi}$, at least one more Hamiltonian parameter must be properly fixed to decouple the additional relevant interaction.

In this paper we investigate this issue by FT methods. We consider the more general theory in which the order parameter $\phi_{a i}$ is an $(N-1) \times 2$ matrix, i.e., $a=1, \ldots N-1$ and $i=1,2$, for $N \geq 3$. In this case, setting $w_{0}=y_{0}=z_{0}=0$ and $r_{\phi}=r_{\psi}$, one recovers the $\mathrm{O}(2) \otimes \mathrm{O}(N)$-symmetric LGW Hamiltonian (1.2). This theory has a stable FP with attraction domain in the region $g_{2,0}>0$ describing a critical behavior with symmetrybreaking pattern $\mathrm{O}(2) \otimes \mathrm{O}(N) \rightarrow \mathrm{O}(2) \otimes \mathrm{O}(N-2)[14,19,20]$. We should mention that the existence of this $\mathrm{FP}$ has been a controversial issue for quite a long time; the different scenarios 
are reviewed in Refs. [2,20-23].

In order to determine the RG flow of the theory (1.3) in three dimensions and determine its multicritical behavior, we consider the minimal-subtraction $(\overline{\mathrm{MS}})$ scheme without $\epsilon$ expansion (henceforth indicated as $3 d$ - $\overline{\mathrm{MS}}$ scheme) in which no $\epsilon$ expansion is performed and $\epsilon$ is set to the physical value $\epsilon=1$ [24]. We use a symbolic manipulation program that generates the diagrams and computes symmetry and group factors, and the compilation of Feynman integrals of Ref. [25]. This allows us to compute the $3 d-\overline{\mathrm{MS}} \beta$-functions to five loops in the full model. The perturbative series are used to determine the FP structure and, in particular, to investigate the existence of stable FPs that may describe the critical behavior at the multicritical point. The $3 d-\overline{\mathrm{MS}}$ scheme is particularly convenient for the three-dimensional FT study of the multicritical behavior. Indeed, the multicritical theory is simply obtained by setting $r_{\phi}=r_{\psi}=0$, i.e. by considering the massless theory. Note that this is not correct in the three-dimensional massive zero-momentum (MZM) scheme [26], in which a proper tuning of $r_{\phi}$ and $r_{\psi}$ is needed.

We summarize the main results of this paper. In order to check the hypothesis of the effective enlargement of the symmetry

$$
\mathrm{O}(2) \otimes\left[\mathbb{Z}_{2} \oplus \mathrm{O}(N-1)\right] \rightarrow \mathrm{O}(2) \otimes \mathrm{O}(N)
$$

for generic $N$-component systems at the multicritical point, we study the stability properties of the $\mathrm{O}(2) \otimes \mathrm{O}(N)$ chiral $\mathrm{FP}$ with respect to all quadratic and quartic perturbations that are symmetric under the reduced symmetry $\mathrm{O}(2) \otimes\left[\mathbb{Z}_{2} \oplus \mathrm{O}(N-1)\right]$. The analysis of the corresponding five-loop series does not support the stability of the $\mathrm{O}(2) \otimes \mathrm{O}(N)$ chiral FP for any value $N \geq 4$, with increasing confidence as $N$ increases. For $N=3$ the results are not conclusive. Our FT results do not allow us to establish the stability properties of the $\mathrm{O}(2) \otimes \mathrm{O}(3) \mathrm{FP}$, which may be either stable or unstable. In the former case, the multicritical behavior would be controlled by the $\mathrm{O}(2) \otimes \mathrm{O}(3) \mathrm{FP}$ if the transition is continuous. In the latter case, we note that the crossover exponent should be very small, $\phi_{4,4} \lesssim 0.1$. Therefore, if the effective quartic Hamiltonian parameters that break the $\mathrm{O}(2) \otimes \mathrm{O}(3)$ symmetry are small, the crossover from the preasymptotic $\mathrm{O}(2) \otimes \mathrm{O}(3)$ critical behavior to the eventual asymptotic behavior is expected to be very slow, and one may observe an effective $\mathrm{O}(2) \otimes \mathrm{O}(3)$ critical behavior for a wide range of reduced-temperature values. We also perform a general PadéBorel analysis of the RG flow for $N=3$, to investigate the existence of stable FPs for generic values of the quartic couplings. No evidence of additional stable FPs is obtained. Therefore, according to our FT results, for $N=3$ the multicritical transition is either controlled by the $\mathrm{O}(2) \otimes \mathrm{O}(3) \mathrm{FP}$ or is of first order.

The paper is organized as follows. In Sec. II we show how the general Hamiltonian (1.3), that has been written down on the basis of symmetry considerations, can be recovered from Hamiltonian (1.1) of easy-axis STAs in a magnetic field along the easy axis. Then, we discuss the mean-field phase diagram, showing that, beside the tetracritical behavior that was predicted in Ref. [10], the model also admits a bicritical and a pentacritical phase diagram. In Sec. III we focus on some particular cases. In Sec. III B we discuss the stability of the $\mathrm{O}(2) \otimes \mathrm{O}(N)$ FPs, and, in Sec. III C, the stability of the decoupled $[\mathrm{O}(2) \otimes \mathrm{O}(N-1)] \oplus \mathrm{O}(2)$ FPs. In Sec. IV we discuss the full model: in Sec. IV A we consider the one-loop $\epsilon$ expansion, while in Sec. IV B we numerically investigate the $\mathrm{RG}$ flow for $N=3$. In Sec. $\mathrm{V}$ we present 
our conclusions and critically discuss the experimental results in view of our findings. In App. A we report a discussion of the mean-field diagram, in App. B we classify all quadratic and quartic perturbations of the $\mathrm{O}(2) \otimes \mathrm{O}(N)$ FPs that are $\mathrm{O}(2)$-invariant. Finally, in App. C we compute the RG dimensions of all quadratic perturbations of the $\mathrm{O}(2) \otimes \mathrm{O}(N)$ symmetric theory.

\section{DERIVATION OF THE GENERAL HAMILTONIAN AND MEAN-FIELD ANALYSIS}

In this Section we derive the effective LGW Hamiltonian (1.3) for easy-axis STAs in a magnetic field along the easy axis described by Hamiltonian (1.1). It can be obtained as usual by first performing a Hubbard-Stratonovitch transformation $[15,16]$. If $\Phi_{a}, a=1,2,3$, is an unconstrained three-component field, the partition function can be rewritten as

$$
Z \sim \int \prod_{a} d \Phi_{a} \exp \left[\frac{1}{2 \beta} \sum_{i j}\left(J^{-1}\right)_{i j} \Phi_{i} \cdot \Phi_{j}-\sum_{i} W\left(\Phi_{i}\right)\right],
$$

where

$$
W(\Phi)=-\ln \left[\frac{\int d^{3} s \delta\left(s^{2}-1\right) \exp \left(\Phi \cdot s-D s_{z}^{2}-H s_{z}\right)}{\int d^{3} s \delta\left(s^{2}-1\right) \exp \left(-D s_{z}^{2}-H s_{z}\right)}\right]
$$

As usual, we now expand $W(\Phi)$ in powers of $\Phi$. At order $\Phi^{4}$ we can write the expansion as

$$
\begin{aligned}
W(\Phi)= & a_{11} \Phi_{z}+a_{21}\left(\Phi_{x}^{2}+\Phi_{y}^{2}\right)+a_{22} \Phi_{z}^{2}+a_{31} \Phi_{z}^{3}+a_{32} \Phi_{z}\left(\Phi_{x}^{2}+\Phi_{y}^{2}\right) \\
& +a_{41}\left(\Phi_{x}^{2}+\Phi_{y}^{2}\right)^{2}+a_{42}\left(\Phi_{x}^{2}+\Phi_{y}^{2}\right) \Phi_{z}^{2}+a_{43} \Phi_{z}^{4}+O\left(\Phi^{5}\right)
\end{aligned}
$$

where $a_{i j}$ can be easily computed in terms of $H$ and $D$. Finally, we identify the critical modes that are associated with the wavevectors $Q$ that maximize the kinetic term. In our case the relevant modes are associated with the wavevector $Q=(4 \pi / 3,0, \pi)$, so that we can write

$$
\begin{aligned}
& \Phi_{x}(r)=c\left[\phi_{11}(r) \cos (Q \cdot r)+\phi_{12}(r) \sin (Q \cdot r)\right], \\
& \Phi_{y}(r)=c\left[\phi_{21}(r) \cos (Q \cdot r)+\phi_{22}(r) \sin (Q \cdot r)\right], \\
& \Phi_{z}(r)=c\left[\psi_{1}(r) \cos (Q \cdot r)+\psi_{2}(r) \sin (Q \cdot r)\right],
\end{aligned}
$$

where we have introduced new fields $\phi_{a i}$ and $\psi_{i}$, with $a=1,2$ and $i=1,2$. The constant $c$ is fixed so that, keeping only slowly varying contributions, we have

$$
\frac{1}{2 \beta} \sum_{i j}\left(J^{-1}\right)_{i j} \Phi_{i} \cdot \Phi_{j} \approx-\int d^{3} x\left[\frac{1}{2} \sum_{\mu}\left[\sum_{a i}\left(\partial_{\mu} \phi_{a i}\right)^{2}+\sum_{i}\left(\partial_{\mu} \psi_{i}\right)^{2}\right]+\frac{1}{2} a_{0}\left(\phi^{2}+\psi^{2}\right)\right] .
$$

Substituting expressions (2.4) in Eq. (2.3) and keeping only slowly varying contributions, we obtain the Hamiltonian (1.3) with $r_{\phi}=\left(c^{2} a_{21}+a_{0}\right), r_{\psi}=\left(c^{2} a_{22}+a_{0}\right), u_{0}=9 a_{42} c^{4} / 2$, $v_{0}=3 a_{42} c^{4}, w_{0}=9\left(2 a_{41}-a_{42}\right) c^{4} / 2, y_{0}=9\left(2 a_{43}-a_{42}\right) c^{4} / 2$, and $z_{0}=3\left(2 a_{41}-a_{42}\right) c^{4}$. This 
derivation gives relations among the different couplings appearing in Eq. (1.3). However, they should not be taken seriously, since the effective Hamiltonian (1.3) is only an approximation of the original one. Note that the odd powers of $\Phi$ present in Eq. (2.3) do not contribute to the effective Hamiltonian. Indeed, here the basic ingredient is the anisotropy, i.e. the breaking of the $\mathrm{O}(3)$ spin symmetry to $\mathbb{Z}_{2} \oplus \mathrm{O}(2)$. The additional breaking of the $\mathbb{Z}_{2}$ symmetry caused by the magnetic field does not play any role, apart from modifying the explicit expressions of the parameters of the LGW Hamiltonian.

Several comments should be made on the derivation of the effective Hamiltonian (1.3). First, we have assumed here that the relevant modes can be inferred from the analysis of the hopping term. While this is correct for unfrustrated systems, for frustrated ones as is the case here the method is questionable and it is indeed possible that some low-temperature properties are not correctly described by this approach [27]. Second, note that the explicit value of $Q$ does not play any role. This means that the effective Hamiltonian (1.3) can be used to describe any critical behavior with a three-component order parameter $S(q)$, with $q \neq K / 2$, where $K$ is a reciprocal-lattice vector. The effective Hamiltonian (1.3) differs from that presented in Refs. $[10,12]$, in which the quartic breaking terms proportional to $w_{0}, y_{0}$, and $z_{0}$ are absent. ${ }^{1}$ In any case, even if absent in the microscopic model, these additional terms would be generated by RG transformations.

The phase diagram of the theory with Hamiltonian (1.3) can be studied within the meanfield approximation. The general discussion is presented in App. A. Here we only report the final results for the specific case in which one of the transitions is a chiral transition, i.e. it is associated with the symmetry-breaking pattern $\mathrm{O}(2) \otimes \mathrm{O}(2) \rightarrow \mathrm{O}(2)_{\text {diag }}$, as it is of interest for easy-axis materials. This occurs when $v_{0}+z_{0}>0$. The reader interested in systems with a collinear/paramagnetic transition is referred to App. A. The possible phase diagrams are reported in Fig. 2. There are three possibilities: (a) a pentacritical point, (b) a tetracritical point, (c) a bicritical point. In all cases but one the transitions are second-order ones; one transition line is of first order. The known easy-axis materials, like $\mathrm{ANiX}_{3}$, with $\mathrm{A}=\mathrm{Cs}$, $\mathrm{Rb}$, and $\mathrm{X}=\mathrm{Cl}, \mathrm{Br}$, or $\mathrm{CsMnI}_{3}$, all show a tetracritical point, i.e. a phase diagram of type (b). This fact should be related to the smallness of the easy-axis anisotropy. Indeed, for $D \rightarrow 0$ we also have $H \rightarrow 0$ at the multicritical point. Thus, the breaking of the $\mathrm{O}(3)$ invariance is expected to be small at the multicritical point, and we can generically assume that $w_{0} \approx y_{0} \approx z_{0} \approx 0$. In this specific case, the mean-field analysis predicts a tetracritical phase diagram irrespective of $u_{0}$ and $v_{0}$, in agreement with experiments. Bicritical behavior is expected for $|D|$ large enough: for $|D|>3 J^{\prime}$, where $J^{\prime}$ is the intraplane coupling, the basal spin components $s_{x}$ and $s_{y}$ should not magnetize at any temperature [1] and thus phase 4 should not occur, forbidding a tetracritical behavior. For $H=0$, these systems should behave as Ising antiferromagnets. Note that, in this case, beside the XY transition predicted by the phase diagram (c), other transitions (probably first-order ones) may occur as $T$ is lowered [28-30]. It is interesting to note that the mean-field analysis also predicts

\footnotetext{
${ }^{1}$ Hamiltonian (1.3) is recovered by adding in the Ansatz of Ref. [10] for the free energy, see their Eq. (3), terms proportional to $\left|S_{z}\right|^{4}, S^{2}\left|S_{z}\right|^{2}$, and $\left(S_{z}^{2} S^{*} \cdot S^{*}+\left(S_{z}^{*}\right)^{2} S \cdot S\right)$. These terms are a priori expected in the presence of easy-axis anisotropy on the basis of symmetry considerations.
} 
(a)

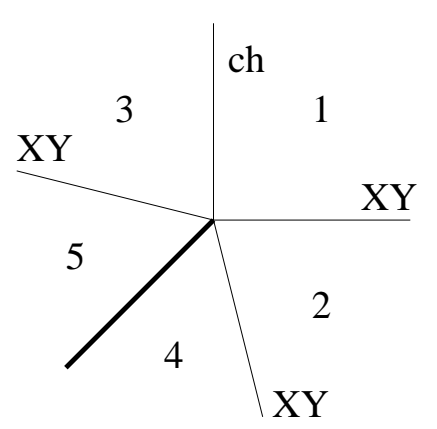

(b)

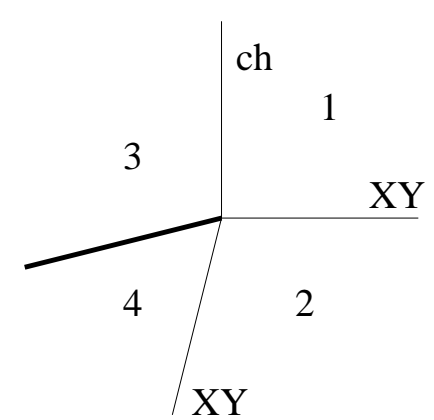

(c)

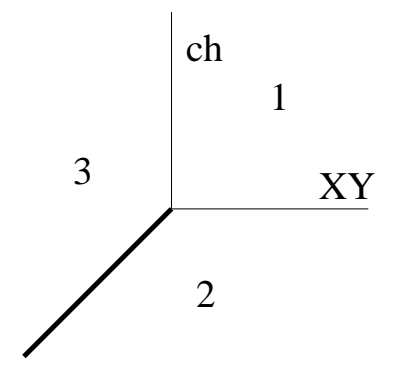

FIG. 2. The possible phase diagrams in the $\left(r_{\phi}, r_{\psi}\right)$ plane predicted by the mean-field approximation with a chiral ("ch") transition. Thin lines represent second-order transitions, while thick lines are first-order transitions. Phase 1 is paramagnetic, in phase $2 \psi \neq 0$ and $\phi=0$, in phase 3 $\psi=0, \phi_{1} \neq 0, \phi_{2} \neq 0$ with $\phi_{1} \cdot \phi_{2}=0$, in phase $4 \phi_{1} \neq 0, \phi_{2}=0, \psi \neq 0$, in phase 5 all vectors are nonvanishing.

a pentacritical point. In this case there is a new phase (phase 5 in Fig. 2) in which the basal spin components $s_{x}$ and $s_{y}$ show a distorted $120^{\circ}$ structure, while the $s_{z}$ component is modulated as in phase 2. The possibility of a tetracritical phase diagram was already noted in Ref. [10]. However, in their work, since they only considered the case $w_{0}=y_{0}=z_{0}=0$, this was the only possible phase diagram. Indeed, the pentacritical and the bicritical points are only obtained if $w_{0}, y_{0}$, and $z_{0}$ are not all vanishing.

\section{ANALYSIS OF SOME PARTICULAR CASES}

\section{A. Particular models and fixed points}

The three-dimensional properties of the RG flow are determined by its FPs. Some of them can be identified by considering particular cases in which some of the quartic parameters vanish. For example, we can easily recognize:

(a) the $\mathrm{O}(K)$-symmetric model is recovered by setting $r_{\phi}=u_{0}=v_{0}=w_{0}=z_{0}=0(K=$ 2), $r_{\psi}=u_{0}=v_{0}=y_{0}=z_{0}=0(K=2 N-2), r_{\phi}=r_{\psi}$ and $v_{0}=w_{0}=y_{0}=z_{0}=0$ $(K=2 N)$. Results for these theories are reviewed, e.g., in Ref. [22]. 
(b) the $\mathrm{O}(2) \otimes \mathrm{O}(K)$ model, cf. Eq. (1.2) with $\Phi_{a i}$ being a $K \times 2$ matrix, for $r_{\phi}=r_{\psi}$ and $w_{0}=y_{0}=z_{0}=0(K=N)$, and for $r_{\psi}=0$ and $u_{0}=v_{0}=y_{0}=0(K=$ $N-1)$. The properties of these models are reviewed in Refs. [2,20,22,23,31]. In three dimensions perturbative calculations within the MZM scheme [19,32] and within the $3 d$ - $\overline{\mathrm{MS}}$ scheme [20] indicate the presence of a stable FP with attraction domain in the region $g_{2,0}>0$ for all values of $K$, except possibly $K=6$. For $K=2$, these conclusions have been recently confirmed by a Monte Carlo calculation [20]. On the other hand, near four dimensions, a stable FP is found only for large values of $K$, i.e., $K>K_{c}=21.80-23.43 \epsilon+7.09 \epsilon^{2}+O\left(\epsilon^{3}\right)[14,33-35]$. A stable FP with attraction domain in the region $g_{2,0}<0$ exists for $K=2$ (it belongs to the XY universality class) [14], for $K=3$ (Ref. [31]), and, as we shall discuss below, for $K=4$. Note that nonperturbative approximate RG calculations have so far found no evidence of stable FPs for $K=2$ and $3[23,36]$. In the following we will call the FP with $g_{2}>0$ chiral $\mathrm{FP}$, while the FP with $g_{2}<0$ will be named collinear FP.

(c) the $\mathrm{O}(2) \oplus \mathrm{O}(K)$ model with $K=2 N-2$ for $v_{0}=z_{0}=0$. This theory describes the multicritical behavior of a model with two order parameters that is symmetric under the group $\mathrm{O}(2) \oplus \mathrm{O}(K)$ [17]. In the case we are interested in, i.e. for $N \geq 3$ and therefore $K \geq 4$, the stable FP is the decoupled FP, corresponding to $u_{0}=0$, which describes a critical behavior in which the two order parameters $\phi$ and $\psi$ are effectively uncoupled at the multicritical point $[18,37]$.

(d) Decoupled $\mathrm{O}(2) \otimes \mathrm{O}(N-1)$ and $\mathrm{O}(2)$ models for $u_{0}=v_{0}=0$. The corresponding stable FP describes two effectively decoupled critical behaviors in the $\mathrm{O}(2) \otimes \mathrm{O}(N-1)$ and $\mathrm{O}(2)$ universality classes.

(e) For $v_{0}=0$ we obtain a multicritical theory with the larger symmetry group $[\mathrm{O}(2) \otimes \mathrm{O}(N-1)] \oplus \mathrm{O}(2)$.

The FPs of the above-mentioned particular models are also FPs of the enlarged model (1.3). Their stability in the full theory can be checked by computing the RG dimensions of the additional terms present in the complete Hamiltonian (1.3).

The analysis of model (1.3) is simplified by the following symmetry transformation. If we transform the fields as

$$
\psi_{i} \rightarrow \sum_{j} \epsilon_{i j} \psi_{j}, \quad \phi_{a i} \rightarrow \phi_{a i}
$$

and the couplings according to

$$
u_{0} \rightarrow u_{0}-v_{0}, \quad v_{0} \rightarrow-v_{0}, \quad w_{0} \rightarrow v_{0}+w_{0}, \quad y_{0} \rightarrow v_{0}+y_{0}, \quad z_{0} \rightarrow 2 v_{0}+z_{0},
$$

we reobtain the same Hamiltonian. This transformation leaves invariant the model with $v_{0}=0$ and maps any model with $v_{0}<0$ to a model with $v_{0}>0$. The transformation (3.2) implies several symmetry properties for the RG functions. In particular, under the transformation of the renormalized quartic couplings 


$$
u \rightarrow u-v, \quad v \rightarrow-v, \quad w \rightarrow v+w, \quad y \rightarrow v+y, \quad z \rightarrow 2 v+z,
$$

the RG functions associated with the exponents are unchanged, while the $\beta$-functions transform covariantly. As a consequence, each $\mathrm{FP}$ with nonvanishing quartic coupling $v<0$ is mapped into another FP with $v>0$ and the same stability properties. The symmetry (3.2) implies that there exists another $\mathrm{O}(2) \otimes \mathrm{O}(N)$ model beside that reported at point (b) above:

$\left(\mathrm{b}^{\prime}\right)$ The $\mathrm{O}(2) \otimes \mathrm{O}(N)$ model is also obtained for $r_{\phi}=r_{\psi}, w_{0}=-v_{0}, y_{0}=-v_{0}, z_{0}=-2 v_{0}$; in this case $g_{1,0}=u_{0}-v_{0}, g_{2,0}=-v_{0}$.

Because of symmetry (3.3) it is enough to study the RG flow for $v \geq 0$.

The results for models (a), (b), ( $\left.b^{\prime}\right),(c)$, and (d) allow us to identify four possible FPs that are candidates for being stable FPs of the full theory. In the $\mathrm{O}(2) \otimes \mathrm{O}(N)$ model (1.2) there may be, depending on the value of $N$, two FPs. The chiral FP is located at $g_{1}=g_{1, \mathrm{ch}}^{*}(N)$ and $g_{2}=g_{2, \mathrm{ch}}^{*}(N)$ with $g_{2, \mathrm{ch}}^{*}(N)>0$ and exists for any value of $N$ except possibly $N=6$ [20]. The collinear FP is located at $g_{1}=g_{1, \mathrm{cl}}^{*}(N)$ and $g_{2}=g_{2, \mathrm{cl}}^{*}(N)$ with $g_{2, \mathrm{cl}}^{*}(N)<0$. Such a FP exists for $N=2[14]$ - in this case it is equivalent to the standard XY FP-and for $N=3$ [31]. We have investigated if the collinear FP exists also for larger values of $N$, by extending the analysis of Ref. [31]. In the $3 d-\overline{\mathrm{MS}}$ scheme at five loops, we find a stable FP only for $N=4: g_{1, \mathrm{cl}}^{*}(4)=0.10(7)$ and $g_{2, \mathrm{cl}}^{*}(4)=-1.83(10){ }^{2}$ For $N=5$, we find a FP only in $1 / 3$ of the cases that are considered and such a percentage decreases as $N$ increases. In the MZM scheme at six loops a FP is found for $N=4,5$, and 6 , and disappears for $N \geq 8$. For $N=4$ it is located at $g_{1, \mathrm{cl}}^{*}(4)=6.1(4)$ and $g_{2, \mathrm{cl}}^{*}(4)=-50(2)$; for $N=5$ and $N=6$ at $g_{1, \mathrm{cl}}^{*}=-7.2(5)$ and $g_{2, \mathrm{cl}}^{*}=-53(3), g_{1, \mathrm{cl}}^{*}=-8.5(7)$ and $g_{2, \mathrm{cl}}^{*}=-56(4)$, respectively. The perturbative analysis provides therefore strong evidence for the existence of a collinear FP for $2 \leq N \leq 4$. For $N \geq 8$ this $\mathrm{FP}$ is absent (in agreement with the large- $N$ analysis), while in the intermediate cases $5 \leq N \leq 7$ it is not clear whether the collinear FP really exists since the two perturbative schemes give opposite results. We have also verified the stability of the collinear FPs within the $\mathrm{O}(2) \otimes \mathrm{O}(N)$ theory $(1.2)$ : whenever they exist, they are stable. However, for $N=4$ we have been unable to estimate the stability eigenvalues. Indeed, the $3 d-\overline{\mathrm{MS}}$ scheme predicts complex stability eigenvalues, while in the MZM scheme we find complex eigenvalues only in $50 \%$ of the cases.

The collinear FP has $g_{2}<0$. Therefore, if we are only interested in the full model for $v \geq 0$, we must consider the FP appearing in model $\left(\mathrm{b}^{\prime}\right)$. Thus, the above-reported results for the $\mathrm{O}(2) \otimes \mathrm{O}(N)$ predict two possible FPs:

(a) $u=g_{1, \mathrm{ch}}^{*}(N), v=g_{2, \mathrm{ch}}^{*}(N), w=y=z=0$;

\footnotetext{
${ }^{2}$ In the $3 d-\overline{\mathrm{MS}}$ scheme the couplings are normalized so that $g_{i}=g_{i, 0} \mu^{-\epsilon} / A_{d}$ with $A_{d}=$ $2^{d-1} \pi^{d / 2} \Gamma(d / 2)$. In the MZM scheme the renormalized couplings $g_{1}$ and $g_{2}$ are normalized so that $g_{i}=g_{i, 0} / m$ at tree level, where $m$ is the renormalized mass. For reference, we report the collinear FP for $N=3$ in these normalizations (Ref. [31]): $g_{1, \mathrm{cl}}^{*}=-7.0(5)$ and $g_{2, \mathrm{cl}}^{*}=-50(2)$ in the MZM scheme, $g_{1, \mathrm{cl}}^{*}=0.04(8)$ and $g_{2, \mathrm{cl}}^{*}=-1.71(9)$ in the $3 d-\overline{\mathrm{MS}}$ scheme. The same normalization is used for the $\overline{\mathrm{MS}}$ couplings in Sec. IV A.
} 
(b) $u=g_{1, \mathrm{cl}}^{*}(N)-g_{2, \mathrm{cl}}^{*}(N), w=y=z / 2=-v=g_{2, \mathrm{cl}}^{*}(N)$.

Analogously, analysis of the decoupled theory gives two possible FPs:

(c) $u=v=0, w=g_{1, \mathrm{ch}}^{*}(N-1), z=g_{2, \mathrm{ch}}^{*}(N-1), y=g_{\mathrm{XY}}^{*}$;

(d) $u=v=0, w=g_{1, \mathrm{cl}}^{*}(N-1), z=g_{2, \mathrm{cl}}^{*}(N-1), y=g_{\mathrm{XY}}^{*} \cdot$

Here $g_{\mathrm{XY}}^{*}$ is the four-point renormalized coupling of the XY theory (numerical estimates can be found in Ref. [38]). Note that FPs (c) and (d) are also FPs of the theory with $v=0$.

\section{B. Stability of the $\mathbf{O}(2) \otimes O(N)$ fixed points}

In this section we study the stability properties of the two FPs that appear in the $\mathrm{O}(2) \otimes \mathrm{O}(N)$ model and, therefore, we check the possibility of an enlargement of the symmetry at the multicritical point from $\mathrm{O}(2) \otimes\left[\mathbb{Z}_{2} \oplus \mathrm{O}(N-1)\right]$ to $\mathrm{O}(2) \otimes \mathrm{O}(N)$. For this purpose we need to classify the perturbations that break the $\mathrm{O}(N)$ symmetry to $\mathrm{O}(N-1)$ and preserve the $\mathrm{O}(2)$ symmetry, according to their transformation properties under the $\mathrm{O}(N)$ group and the number of fields. This is done in detail in App. B. The multicritical Hamiltonian (1.3) can be rewritten as

$$
\mathcal{H}=\mathcal{H}_{\text {sym }}+r_{2} V^{(2,2)}+f_{1} V^{(4,4)}+f_{2} V^{(4,2,1)}+f_{3} V^{(4,2,2)},
$$

where $\mathcal{H}_{\text {sym }}$ is the $\mathrm{O}(2) \otimes \mathrm{O}(N)$-symmetric Hamiltonian (1.2) obtained by setting $w_{0}=y_{0}=$ $z_{0}=0$ and $r_{\psi}=r_{\phi}$, and $V^{(2,2)}, V^{(4,4)}, V^{(4,2,1)}$, and $V^{(4,2,2)}$ are respectively a spin-2 quadratic term, a spin-4 quartic term, and two spin-2 quartic terms. Their explicit expressions can be found in App. B. The spin-2 quadratic pertubation $V^{(2,2)}=\phi^{2} / N-(N-1) \psi^{2} / N$ is always relevant. Its $\mathrm{RG}$ dimension at the $\mathrm{O}(2) \otimes \mathrm{O}(N)$ FP gives the crossover exponent, i.e. $\phi=\nu y_{2,2}$, where $\nu$ is the correlation-length exponent.

Let us first discuss the chiral FP (a) that has $g_{2, \mathrm{ch}}^{*}(N)>0$. The exponent $y_{2,2}$ coincides with the exponent $y_{3}$ defined in App. C, since $V^{(2,2)}=\sum_{k=1}^{N-1} O_{k k}^{(3)}$. For $N=3$, we obtain $y_{2,2}=1.49(3)$ in the MZM scheme and $y_{2,2}=1.54(8)$ in the $3 d$ - $\overline{\mathrm{MS}}$ scheme.

In order to estimate the RG dimensions $y_{4,2,1}, y_{4,2,2}$, and $y_{4,4}$ of the quartic perturbations, we computed the corresponding five-loop series in the $\overline{\mathrm{MS}}$ scheme and we analyzed them within the $3 d-\overline{\mathrm{MS}}$ scheme. Note that, since the spin-2 quartic operators mix, $y_{4,2,1}$ and $y_{4,2,2}$ are the eigenvalues of the corresponding RG-dimension matrix. We also estimated the spin$4 \mathrm{RG}$ dimension $y_{4,4}$ by computing the corresponding five-loop series in the MZM scheme. In this scheme we cannot estimate the RG dimensions of the spin-2 quartic perturbations because they also mix with the lower-dimension spin-2 operator $V_{2,2}$. Such a mixing does not occur in the $3 d$ - $\overline{\mathrm{MS}}$ scheme, since in this case the theory is massless and, therefore, operators of different naive dimensions do not mix under renormalization.

Here we only report the series for $y_{4,4}$, which will be the most relevant for the analysis of the stability of the $\mathrm{O}(2) \otimes \mathrm{O}(N) \mathrm{FP}$, for $N=3$ and 4 , and in the $\overline{\mathrm{MS}}$ and MZM schemes. In the $\overline{\mathrm{MS}}$ scheme we have

$$
y_{4,4}=\epsilon+\sum_{i j} a_{i j} g_{1}^{i} g_{2}^{j}
$$


TABLE I. Coefficients $a_{i j}$ of the five-loop $\overline{\mathrm{MS}}$ and MZM expansions of $y_{4,4}$, cf. Eqs. (3.5) and (3.6), for $N=3$ and 4 .

\begin{tabular}{|c|c|c|c|c|}
\hline \multirow[b]{2}{*}{$i, j$} & \multicolumn{2}{|c|}{$\overline{\overline{\mathrm{MS}}}$} & \multicolumn{2}{|c|}{ MZM } \\
\hline & $N=3$ & $N=4$ & $N=3$ & $N=4$ \\
\hline 1,0 & -2 & -2 & $-6 / 7$ & $-3 / 4$ \\
\hline 0,1 & $-1 / 3$ & $-1 / 3$ & $-2 / 9$ & $-2 / 9$ \\
\hline 2,0 & $28 / 9$ & $61 / 18$ & 0.383976 & 0.320602 \\
\hline 1,1 & $1 / 9$ & $-1 / 6$ & 0.018812 & -0.030864 \\
\hline 0,2 & $-1 / 36$ & $1 / 8$ & -0.005487 & 0.041152 \\
\hline 3,0 & -11.157290 & -12.122093 & -0.246143 & -0.176781 \\
\hline 2,1 & -0.245370 & 0.984242 & -0.016559 & 0.015113 \\
\hline 1,2 & -1.174531 & -2.872196 & -0.072221 & -0.142869 \\
\hline 0,3 & 0.824994 & 1.509014 & 0.080442 & 0.140829 \\
\hline 4,0 & 62.535697 & 72.040868 & 0.225243 & 0.152346 \\
\hline 3,1 & -6.135544 & -19.807535 & -0.038487 & -0.083599 \\
\hline 2,2 & 14.290714 & 31.675613 & 0.157437 & 0.252196 \\
\hline 1,3 & -8.768847 & -16.889435 & -0.138290 & -0.224249 \\
\hline 0,4 & 0.999971 & 1.877179 & 0.008548 & 0.019106 \\
\hline 5,0 & -422.234940 & -508.920947 & -0.231515 & -0.138376 \\
\hline 4,1 & 90.411170 & 230.058460 & 0.069042 & 0.102807 \\
\hline 3,2 & -166.086612 & -359.525801 & -0.268436 & -0.357188 \\
\hline 2,3 & 108.042417 & 220.680543 & 0.271663 & 0.388611 \\
\hline 1,4 & -23.261600 & -49.094226 & -0.061312 & -0.100610 \\
\hline 0,5 & 2.329711 & 4.789714 & 0.015950 & 0.019262 \\
\hline
\end{tabular}

with $\epsilon=1$ in three dimensions. The coefficients $a_{i j}$ for $N=3,4$ are reported in Table I to five loops, i.e. for $i+j \leq 5$. The renormalized $\overline{\mathrm{MS}}$ couplings $g_{1}$ and $g_{2}$, corresponding to the quartic parameters $g_{1,0}$ and $g_{2,0}$ of the Hamiltonian (1.2), are normalized as in Ref. [20], see footnote 2 . In the MZM scheme we have the analogous expansion ${ }^{3}$

$$
y_{4,4}=1+\sum_{i j} a_{i j} \bar{g}_{1}^{i} \bar{g}_{2}^{j}
$$

The coefficients $a_{i j}$ to five loops are reported in Table I for $N=3,4$. The renormalized MZM couplings $\bar{g}_{1,2}$ are normalized as in Ref. [19], i.e. $\bar{g}_{i} \approx c_{i} g_{i, 0} / m$ at tree order, where $c_{1}=(8+N) /(48 \pi)$ and $c_{2}=3 /(16 \pi)$. The perturbative series that are not reported here are available on request.

We analyzed the series using the conformal-mapping method and the Padé-Borel method, following closely Refs. [40,20], to which we refer for details. The error on the conformalmethod results takes into account the spread of the results as the parameters $\alpha$ and $b$ are varied (cf. Ref. [40] for definitions) and the error due to the uncertainty of the FP location (we use the estimates reported in Refs. [19,32,20,31]). In the Padé-Borel analysis we used the Padé [4/1] and several values of the parameter $b$. Again, the error takes into account the dependence on $b$ and the uncertainty of the FP location. In the Padé-Borel analyses of $y_{4,4}$ the error also takes into account the difference between the [4/1] and the [3/1] estimates. This was not done for the spin-2 RG dimensions since in that case the results obtained by

\footnotetext{
${ }^{3}$ The perturbative MZM series were obtained by using a symbolic manipulation program that generated the diagrams, the symmetry, and group factors. Numerical estimates of the Feynman integrals were taken from Ref. [39].
} 
TABLE II. Estimates of the RG dimensions $y_{4,2,1}, y_{4,2,2}$, and $y_{4,4}$ of the operators $V^{(4,2,1)}$, $V^{(4,2,2)}$, and $V^{(4,4)}$ at the chiral (ch) FP and at the collinear (cl) FP. Results have been obtained in the $3 d-\overline{\mathrm{MS}}$ scheme $(\overline{\mathrm{MS}})$ and in the three-dimensional massive zero-momentum scheme (MZM). For the resummation of the five-loop perturbative series the conformal-mapping (CM) and the Padé-Borel (PB) methods have been used. For $y_{4,4}$ two estimates are reported in each case, resspectively from the analysis of the series of $y_{4,4}$ and $1 / y_{4,4}$.

\begin{tabular}{|c|c|c|c|c|c|c|c|}
\hline \multirow[t]{2}{*}{$\mathrm{FP}, N$} & \multicolumn{2}{|c|}{$y_{4,2,1}$} & \multicolumn{2}{|c|}{$y_{4,2,2}$} & \multicolumn{3}{|c|}{$y_{4,4}$} \\
\hline & $\overline{\mathrm{MS}}, \mathrm{CM}$ & $\overline{\mathrm{MS}}, \mathrm{PB}$ & $\overline{\mathrm{MS}}, \mathrm{CM}$ & $\overline{\mathrm{MS}}, \mathrm{PB}$ & $\overline{\mathrm{MS}}, \mathrm{CM}$ & $\overline{\mathrm{MS}}, \mathrm{PB}$ & MZM, CM \\
\hline$\overline{c h, 3}$ & $-0.7(9)$ & $-1.8(7)$ & $0.0(7)$ & $-0.9(6)$ & $-0.4(5), 0.12(5)$ & $-0.4(4), 0.07(3)$ & $-0.3(2), 0.05(2)$ \\
\hline $\operatorname{ch}, 4$ & $-0.7(5)$ & $-1.0(3)$ & $0.3(3)$ & $-0.2(3)$ & $-0.1(2), 0.18(4)$ & $-0.2(2), 0.12(4)$ & $0.15(12), 0.16(4)$ \\
\hline $\operatorname{ch}, 5$ & $-0.8(7)$ & $-0.6(3)$ & $0.2(2)$ & $0.0(2)$ & $0.0(2), 0.22(4)$ & $0.02(11), 0.18(5)$ & $0.3(2), 0.24(5)$ \\
\hline $\operatorname{ch}, 6$ & $-0.6(6)$ & $-0.5(2)$ & $0.25(13)$ & $0.11(15)$ & $0.1(2), 0.26(4)$ & $0.13(10), 0.23(5)$ & \\
\hline $\mathrm{ch}, 8$ & $-0.5(5)$ & $-0.4(2)$ & $0.22(9)$ & $0.17(6)$ & $0.2(2), 0.34(5)$ & $0.29(8), 0.32(4)$ & $0.26(2), 0.29(2)$ \\
\hline $\mathrm{ch}, 16$ & $-0.3(2)$ & $-0.17(6)$ & $0.09(2)$ & $0.08(3)$ & $0.56(4), 0.570(9)$ & $0.60(2), 0.58(3)$ & $0.56(2), 0.54(2)$ \\
\hline $\operatorname{ch}, \infty$ & \multirow{3}{*}{\multicolumn{2}{|c|}{0}} & \multirow{3}{*}{\multicolumn{2}{|c|}{0}} & & 1 & \\
\hline $\mathrm{cl}, 3$ & & & & & $0.3(4), 0.7(2)$ & $-0.7(1.1), 0.5(2)$ & $0.5(1.0), 1.2(7)$ \\
\hline $\mathrm{cl}, 4$ & & & & & $-0.2(8), 0.5(2)$ & $-1(1), 0.35(15)$ & $0.5(1.1), 0.9(3)$ \\
\hline
\end{tabular}

using the Padé [3/1] did not look reliable. They varied significantly with $b$ and, for $N \leq 5$, favored complex estimates of $y_{4,2,1}$ and $y_{4,2,2}$.

The results of the analyses are reported in Table II. We first computed the spin-2 RG dimensions $y_{4,2,1}$ and $y_{4,2,2}$. If $Y_{i j}, j=1,2$, is the anomalous-dimension matrix, $y_{4,2,1}$ and $y_{4,2,2}$ are the eigenvalues of $Y$. In order to determine them, we resummed the elements $Y_{i j}$ and computed the two eigenvalues. The results we report are obtained by averaging the eigenvalues over many different choices of approximants. The results have a quite large error, so that it is impossible to draw definite conclusions on the relevance of these operators. For $N \geq 5$ the results favor $y_{4,2,2}>0$, so that one operator would be relevant, while for $N=3$ it seems likely that the spin-2 perturbations are irrelevant. For the spin-4 RG dimension we report two estimates for each case. They are obtained from the analysis of the series of $y_{4,4}$ and $1 / y_{4,4}$, respectively. Their difference should allow us to estimate systematic errors in the series resummations that are not taken into account by the spread of the approximants, which we usually take as an indication of the error. We find $y_{4,4}>0$ for all $N \geq 4$, with increasing confidence as $N$ increases (for $N=4$ we mainly rely on the MZM analysis that predicts $y_{4,4}>0$ both for the direct and for the inverse series). For $N=3$, the results of the analysis of $y_{44}$ and $1 / y_{44}$ differ substantially (the error of the estimate from $1 / y_{44}$ may be underestimated) and even have opposite signs. This does not allow us to establish whether $V^{(4,4)}$ is a relevant or an irrelevant perturbation of the chiral FP.

Let us briefly discuss the physical picture in the two cases. If the $\mathrm{O}(2) \otimes \mathrm{O}(3) \mathrm{FP}$ is stable, it controls the critical behavior of statistical systems in its attraction domain. Setting $t \equiv\left(T-T_{m c}\right) / T_{m c}$, where $T_{m c}$ is the critical temperature at the multicritical point, the singular part of the free energy can be written as

$$
\mathcal{F}=|t|^{3 \nu} f\left(A|t|^{-\phi}\right)
$$

where $A$ is the scaling field associated with the anisotropy - in STA's it will be a combination of $T, D$, and $H-\nu \approx 0.6$ is the correlation-length exponent of the $\mathrm{O}(2) \otimes \mathrm{O}(3)$ theory $[19,20]$, $\phi=y_{2,2} \nu \approx 0.9$ is the crossover exponent, and $f(x)$ is a scaling function. 
If the $\mathrm{O}(2) \otimes \mathrm{O}(3)$ is unstable with respect to the spin- 4 quartic perturbation, one should also consider the crossover exponent $\phi_{4,4}$ associated with the spin- 4 quartic instability. This is expected to be quite small. Indeed, our results indicate $y_{4,4} \lesssim 0.2$ for $N=3$, so that $\phi_{4,4} \lesssim 0.1$. Therefore, if the $\mathrm{O}(2) \otimes \mathrm{O}(3) \mathrm{FP}$ is unstable and the effective quartic Hamiltonian parameters breaking the $\mathrm{O}(2) \otimes \mathrm{O}(3)$ symmetry are small, the crossover from the preasymptotic $\mathrm{O}(2) \otimes \mathrm{O}(3)$ critical behavior to the eventual asymptotic behavior is expected to be very slow and one may observe an effective $\mathrm{O}(2) \otimes \mathrm{O}(3)$ critical behavior for a wide range of reduced-temperature values.

Now, let us consider the collinear FP (b) that has $g_{2, \mathrm{cl}}^{*}(N)<0$ for $N=3$ and $N=4$. For the spin-2 operators the analysis indicates that they are irrelevant at the FP, but is not precise enough to allow a quantitative determination of $y_{4,2,1}$ and $y_{4,2,2}$. For $N=3$, by using the conformal-mapping method we find that approximately $50 \%$ of the approximants give complex spin-2 RG dimensions (the real part is always negative) and $50 \%$ provide real negative estimates. Padé-Borel approximants always give real negative estimates. The same pattern is observed for $N=4$. The results for the spin- 4 operator are reported in Table II. They suggest that the collinear FP is unstable for both $N=3$ and $N=4$.

In conclusion, the perturbative analysis shows that the collinear FP is always unstable, while the chiral FP is unstable for $N \geq 4$. For $N=3$ the results are not conclusive and the $\mathrm{O}(2) \otimes \mathrm{O}(3)$ chiral FP may be either stable or unstable.

\section{Stability of the decoupled $[\mathrm{O}(2) \otimes \mathbf{O}(N-1)] \oplus \mathbf{O}(2)$ fixed points}

Two other interesting FPs can be investigated by essentially nonperturbative arguments, FPs (c) and (d) discussed in Sec. III A. In order to check the stability of these FPs, we must determine the RG dimensions at the decoupled FPs of the perturbations

$$
P_{E}=\int d^{3} x \phi^{2} \psi^{2}, \quad P_{T}=\int d^{3} x \sum_{i j} O_{i j}^{(4)} T_{i j},
$$

where $O_{i j}^{(4)}$ is defined in App. C, cf. Eq. (C4) and $T_{i j}=\psi_{i} \psi_{j}-\frac{1}{2} \delta_{i j} \psi^{2}$. Simple RG arguments show that the $\mathrm{RG}$ dimension of $P_{E}$, which is an energy-energy term, is given by

$$
y_{E}=\frac{1}{\nu_{\psi}}+\frac{1}{\nu_{\phi}}-3=\frac{\alpha_{\psi}}{2 \nu_{\psi}}+\frac{\alpha_{\phi}}{2 \nu_{\phi}},
$$

where $\alpha_{\psi}$ and $\nu_{\psi}$ are the critical exponents of the 3-dimensional XY universality class $\left(\alpha_{\psi}=-0.0146(8)\right.$ and $\nu_{\psi}=0.67155(26)$, see Ref. [38]), while $\alpha_{\phi}$ and $\nu_{\phi}$ are those of the 3-dimensional theory associated with the $\mathrm{O}(2) \otimes \mathrm{O}(N-1)$ FP. Analogously, for the RG dimension of $P_{T}$ we obtain the relation

$$
y_{T}=y_{T, \psi}+y_{4, \phi}-3,
$$

where $y_{T, \psi}=y_{T, \mathrm{XY}}, y_{T, \mathrm{XY}}$ is RG dimension of the operator $T_{i j}$ at the XY FP $\left(y_{T, \mathrm{XY}} \approx 1.77\right.$ $[41,42])$, and $y_{4, \phi}$ is the RG dimension of the quadratic operator $O_{i j}^{(4)}$ introduced in App. C, computed at the $\mathrm{O}(2) \otimes \mathrm{O}(N-1) \mathrm{FP}$. 
Let us consider first the chiral FP (c) that has $z>0$. Estimates of the exponents $\alpha_{\phi}$ and $\nu_{\phi}$ for several values of $N$ are reported in Refs. [19,32,20], while estimates of $y_{4, \phi}$ are reported in App. C. It is then easy to check that $y_{E}>0$ for $N \lesssim 6$ and $y_{E}<0$ for $N \gtrsim 6$. Therefore, the perturbation $P_{E}$ is relevant for $N \lesssim 6$. Analogously, we obtain $y_{T} \approx 0$ for $N=3$ and $y_{T}<0$ for $N>3$. Thus, except possibly for $N=3, P_{T}$ is always irrelevant. These results indicate that the decoupled FP (c) is unstable for $N \lesssim 6$, and therefore for the interesting case $N=3$.

Let us now consider the collinear FP (d). For $N=3$ the collinear FP in the $\mathrm{O}(2) \otimes \mathrm{O}(2)$ theory belongs to the XY universality class [14]. It is easy to show that $\alpha_{\phi}=\alpha_{\mathrm{XY}}$, which gives $y_{E} \approx-0.04$. Thus $P_{E}$ is irrelevant. As for $P_{T}$, using the results of App. C, we have $y_{4, \phi}=2 y_{h, \mathrm{XY}}-3$, where $y_{h, \mathrm{XY}}=\left(5-\eta_{\mathrm{XY}}\right) / 2$ is the RG dimension of the field in the $\mathrm{XY}$ model. Therefore, in three dimensions $y_{T}=y_{T, \mathrm{XY}}-\eta_{\mathrm{XY}}-1 \approx 0.73$, which means that $P_{T}$ is relevant, and the collinear decoupled FP (d) is unstable. For $N=4$ and $N=5$, using the results of App. C, we have $y_{T}>0$ in both cases. In order to compute $y_{E}$ we determined $\nu_{\phi}$ in the $\mathrm{O}(2) \otimes \mathrm{O}(N-1)$ theory at the collinear FP. In the $\mathrm{O}(2) \otimes \mathrm{O}(3)$ case we have [31] $\nu_{\phi}=0.63(8)$ (3d- $\overline{\mathrm{MS}}$, conformal mapping) and $\nu_{\phi}=0.59(4)$ (MZM, conformal mapping); in the $\mathrm{O}(2) \otimes \mathrm{O}(4)$ case we have $\nu_{\phi}=0.76(9)\left(3 d-\overline{\mathrm{MS}}\right.$, conformal mapping) and $\nu_{\phi}=0.64(7)$ (MZM, conformal mapping). These estimates imply $y_{E}>0$ for $N=4$ and $-0.3 \lesssim y_{E} \lesssim 0.2$ for $N=5$.

In conclusion, the collinear decoupled FP is always unstable, while the chiral decoupled FP is unstable for $N \lesssim 6$, stable in the opposite case.

As we have mentioned in Sec. III A the decoupled FPs are also FPs for the multicritical theory with $v_{0}=0$. The analysis of their stability in this particular case follows from the results reported above. Indeed, for the theory with $v_{0}=0$ it is enough to consider the perturbation $P_{E}$. Thus, the decoupled chiral FP (c) is unstable for $N \lesssim 6$ and stable in the opposite case. The decoupled collinear FP (d) is stable for $N=3$ and possibly for $N=5$.

\section{THE RENORMALIZATION-GROUP FLOW IN THE FULL THEORY}

\section{A. Renormalization-group flow near four dimensions}

In Sec. III we considered some particular FPs, checking whether they were stable in the full theory. In order to investigate the presence of other FPs, we now perform a one-loop $\epsilon$-expansion analysis. The one-loop $\beta$ functions in the $\overline{\mathrm{MS}}$ scheme are:

$$
\begin{aligned}
& \beta_{u}=-\epsilon u+\frac{N+4}{3} u^{2}-\frac{N-1}{3} v\left(u-\frac{v}{2}\right)+w\left(\frac{N}{3} u-\frac{N-1}{6} v\right) \\
& +y\left(\frac{2}{3} u-\frac{v}{6}\right)-z \frac{N-2}{6}(u-v), \\
& \beta_{v}=-\epsilon v+\frac{N-6}{6} v^{2}+2 u v+\frac{w v}{3}+\frac{y v}{3}+\frac{z(N-2)}{6} v \\
& \beta_{w}=-\epsilon w+\frac{N+3}{3} w^{2}-\frac{N-2}{3} z\left(w-\frac{z}{2}\right)+u\left(\frac{N+6}{3} w-\frac{2}{3} y+\frac{2-N}{6} z\right)
\end{aligned}
$$




$$
\begin{gathered}
+v\left(-\frac{N-3}{6} w+\frac{1}{6} y+\frac{N-2}{6} z\right) \\
\beta_{y}=-\epsilon y+\frac{5}{3} y^{2}+u\left(\frac{8}{3} y+\frac{N-2}{6} z-\frac{N}{3} w\right)+v\left(\frac{N-1}{6} w+\frac{y}{6}-\frac{N-2}{6} z\right), \\
\beta_{z}=-\epsilon z+2 w z+\frac{N-7}{6} z^{2}+2 z u+v\left(\frac{5}{3} w-\frac{y}{3}+\frac{N-12}{6} z\right)
\end{gathered}
$$

where $u, v, w, y$, and $z$ are the $\overline{\mathrm{MS}}$ renormalized couplings (see footnote 2 for a precise definition).

For $N<N_{c} \approx 21.8+O(\epsilon)$ one finds only six FPs (they belong to the models considered in Sec. III A) and none of them is stable. At $N=N_{c}$ four new FPs appear: they belong to the $\mathrm{O}(2) \otimes \mathrm{O}(N)$ models considered in Sec. III A [cf. cases (b) and $\left(\mathrm{b}^{\prime}\right)$ ]. It is easy to check that none of them is stable, as it happens in three dimensions, cf. Sec. III B. Therefore, the enlargement of the symmetry to $\mathrm{O}(2) \otimes \mathrm{O}(N)$ is never realized near four dimensions, for any value of $N$. Stable FPs are found only for $N>N_{1} \approx 23.97$. For $N_{1}<N<N_{2} \approx 24.15$ the stable FP has $v=0$ and does not belong to any of the models considered in Sec. III A. For $N>N_{2}$ the only stable FP is the decoupled chiral FP. Note that, since the zeroes of the $\beta$-functions are not degenerate for all $N \geq 2$, no new FP can emerge within the $\epsilon$ expansion at higher order. The conclusions on the stable FPs apply unchanged to the multicritical theory with $v_{0}=0$ since the stable FPs always have $v=0$.

This analysis can be extended to higher orders of the $\epsilon$ expansion, using the five-loop $\overline{\mathrm{MS}}$ series. On the basis of our experience with the $\mathrm{O}(2) \otimes \mathrm{O}(N)$ model we expect this analysis to be reliable only for large $N$. Indeed, in the $\mathrm{O}(2) \otimes \mathrm{O}(N)$ theory the $\epsilon$ expansion is not able to provide the correct three-dimensional scenario for the physically interesting cases $N=2$ and 3, see, e.g., Ref. [20]. Therefore, we only studied the stability of the decoupled chiral $\mathrm{FP}$, that, according to the analysis presented in Sec. III C, should be stable for $N \gtrsim 6$. For this purpose we computed the stability-boundary function $N_{2}(\epsilon) \equiv n_{2}(\epsilon)+1=24.15+O(\epsilon)$. Repeating the analysis of Sec. III C close to four dimensions, it is easy to show that $n_{2}(\epsilon)$ is determined from

$$
\frac{1}{\nu_{\phi}\left(n_{2}, \epsilon\right)}+\frac{1}{\nu_{\psi}(\epsilon)}=4-\epsilon
$$

where $\nu_{\phi}\left(n_{2}, \epsilon\right)$ and $\nu_{\psi}(\epsilon)$ are the exponents for the chiral theory $\mathrm{O}(2) \otimes \mathrm{O}\left(n_{2}\right)$ and for the $\mathrm{XY}$ model, respectively. At five loops we obtain

$$
n_{2}(\epsilon)=23.1513-28.2072 \epsilon+18.8689 \epsilon^{2}-27.1434 \epsilon^{3}+74.2373 \epsilon^{4}+O\left(\epsilon^{5}\right) .
$$

A Padé-Borel analysis of this series gives $N_{2}(1) \approx 7,4,7$, at five, four, and three loops. The estimates nicely oscillate and allow us to predict $4 \lesssim N_{2}(1) \lesssim 7$. We also analyzed the inverse series $1 / n_{2}(\epsilon)$. A Padé-Borel analysis of the series is possible only at five loops; at four loops all Padé approximants are defective. In this case we obtain $N_{2}(1) \approx 8$. These results are fully compatible with the conclusions of Sec. III C. 


\section{B. Renormalization-group flow in the $3 d-\overline{\mathrm{MS}}$ scheme for $N=3$}

In order to investigate the $\mathrm{RG}$ flow in the complete space of the quartic parameters and check for the existence of other stable FPs, we compute the five-loop series of the $\beta$ functions associated with the five quartic couplings in the $\overline{\mathrm{MS}}$ scheme for the general theory (1.3). The diagrams contributing to this calculation are a few hundreds. We handled them with a symbolic manipulation program, which generated the diagrams and computed the symmetry and group factors of each of them. We used the results of Ref. [25], where the primitive divergent parts of all integrals appearing in our computation are reported. The numerical analysis of the series is very complex and long, so that we only study the physical case $N=3$.

To find the stable FPs we follow the RG flow generated by the resummed $\beta$ functions. For the resummation we use the Padé-Borel method, since the large-order behavior of the series, needed to perform the conformal mapping, is not known. We use several different Padé approximants, the [4/1] and the [3/2] approximants at five loops, and the [3/1] and the $[2 / 2]$ at four loops. We consider several initial conditions close to the Gaussian FP, that allow us to explore a large region in the space of the five renormalized couplings. For most of the employed approximants we found runaway trajectories, up to where the resummation is effective. There are however two notable exceptions. First, if we use the [4/1] Padé approximant, we find that the RG flow ends at the chiral $\mathrm{O}(2) \otimes \mathrm{O}(3) \mathrm{FP}$. This is in agreement with the conclusions of Sec. III B: this FP may be the stable FP of the model. Second, at four loops, by using the [3/1] Padé approximant, one observes a stable FP with $v>0$. However, there is no indication for such a FP at five loops, even as an unstable FP.

In conclusion, the Padé-Borel analysis of the $\mathrm{RG}$ flow does not provide any evidence for a stable FP beside the chiral $\mathrm{O}(2) \otimes \mathrm{O}(3) \mathrm{FP}$. Thus, the multicritical transition is either controlled by this $\mathrm{FP}$ or is of first order.

We also considered the multicritical model with $v=0$. The nonperturbative analysis of Sec. IIIC indicates that the decoupled collinear FP is stable for $N=3$. We also analyzed the full model, checking whether other FPs are present. The five-loop analysis did not find additional FPs, so that the multicritical behavior should be controlled by the decoupled collinear FP (of course, when the transition is continuous).

\section{CONCLUSIONS}

In this paper we study a general model with symmetry $\mathrm{O}(2) \otimes\left[\mathbb{Z}_{2} \oplus \mathrm{O}(N-1)\right]$ for $N \geq 3$, focusing on the nature of the multicritical point. For $N \gtrsim 6$, the theory presents a stable FP that is expected to control the multicritical behavior: the decoupled FP where the fields $\phi$ and $\psi$ become separately critical. Their critical fluctuations are controlled respectively by the chiral $\mathrm{O}(2) \otimes \mathrm{O}(N-1) \mathrm{FP}$ and by the XY FP. For $3<N \lesssim 6$ neither the decoupled FP nor the chiral $\mathrm{O}(2) \otimes \mathrm{O}(N) \mathrm{FP}$ are stable. However, we have not performed a thourough analysis of the RG flow, so that we cannot exclude that there exists a nontrivial stable FP that does not belong to any submodel we have investigated. A complete analysis has been performed for the physically interesting case $N=3$. In this case, we find that the only possible stable FP is the chiral $\mathrm{O}(2) \otimes \mathrm{O}(3) \mathrm{FP}$. The perturbative analysis in two different 

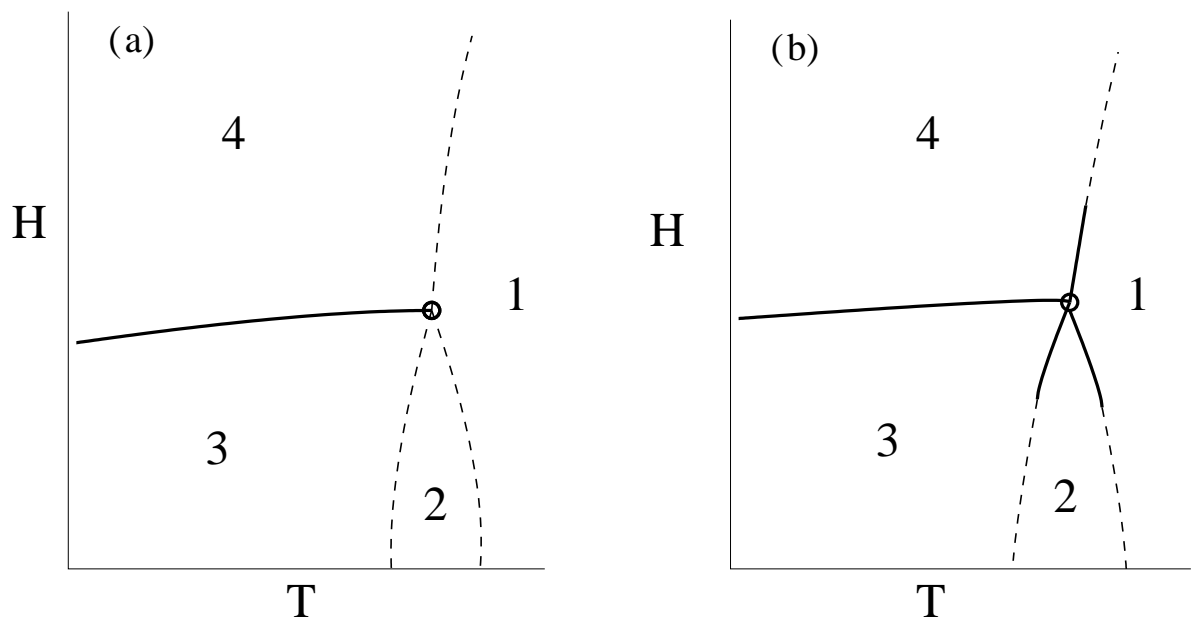

FIG. 3. Possible phase diagrams for easy-axis STAs in a magnetic field: (a) the multicritical transition is continuous; (b) the multicritical transition is of first order. Continuous thick lines represent first-order transitions, while the dashed lines correspond to second-order transitions.

perturbative schemes is unable to draw a definite conclusion on the stability of this FP. The possible phase diagrams are reported in Fig. 3. In phase diagram (a) the transition at the multicritical point is continuous and controlled by the $\mathrm{O}(2) \otimes \mathrm{O}(3) \mathrm{FP}$, thus showing the symmetry enlargement originally put forward in Ref. [12]; this scenario requires the stability of the $\mathrm{O}(2) \otimes \mathrm{O}(3) \mathrm{FP}$ and that the system is within its attraction domain. In the other possible cases, i.e. if the $\mathrm{O}(2) \otimes \mathrm{O}(3) \mathrm{FP}$ is unstable or if the system is outside its attraction domain, we should observe phase diagram (b), in which the multicritical transition is of first order; first-order transitions are also expected along the lines separating phases $1-4,1-2$, and 1-3, close to the multicritical point.

It is interesting to compare this scenario with the experimental results [1]. The behavior observed in experiments at the multicritical point in always compatible with a second-order transition. Moreover, the experimental estimates for the critical exponents (see the results for $\mathrm{CsNiCl}_{3}$ and $\mathrm{CsMnI}_{3}$ in Ref. [1]) are reasonably close to the theoretical results for the $\mathrm{O}(2) \otimes \mathrm{O}(3)$ chiral universality class. Therefore, the phase diagram presented in Fig. 3(a) seems to be favored by experiments, even though the first-order scenario is not necessarily ruled out. If phase diagram (b) is the correct one, a possible explanation of the experiments is that the first-order transition is rather weak, so that all experiments are still probing a crossover region. This interpretation may be supported by the following reasoning. The experimental systems have a small easy-axis anisotropy and therefore, see Sec. II, they are approximately described by the effective theory with $w_{0} \approx y_{0} \approx z_{0} \approx 0$. Thus, the RG flow starts very close to the $\mathrm{O}(2) \otimes \mathrm{O}(3) \mathrm{FP}$, so that one expects strong crossover effects controlled by the chiral $\mathrm{O}(2) \otimes \mathrm{O}(3)$ theory. Moreover, the flow out of the chiral $\mathrm{O}(2) \otimes \mathrm{O}(3) \mathrm{FP}$ should be very slow, since the associated crossover exponent $\phi_{4,4}$ is very small, $\phi_{4,4} \lesssim 0.1$. The firstorder nature of the multicritical point also implies that along the lines separating phases 1-2 and 2-3, one should observe first-order transitions, a tricritical point, and then XY behavior. The presence of the tricritical point might explain why at the transitions for $H=0$ one observes values of $\nu$ and $\gamma$ that are significantly different from the XY estimates and that are closer to the mean-field predictions (see the results for $\mathrm{CsMnI}_{3}$ reported in Ref. [43]). 
Finally, the presence of the first-order transition might also explain the discrepancies between the experimental estimates of the critical exponents along the line 1-4 and the theoretical predictions for the $\mathrm{O}(2) \otimes \mathrm{O}(2)$ chiral universality class. Indeed, as discussed in Ref. [20], close to the first-order parameter region, $\mathrm{O}(2) \otimes \mathrm{O}(2)$ chiral systems show strong crossover effects, with effective exponents that may significantly differ from their asymptotic value.

\section{APPENDIX A: MEAN-FIELD PHASE DIAGRAM}

\section{Model with $v_{0}=0$}

We begin by discussing the mean-field phase diagram for $v_{0}=0$, which corresponds to the model with larger symmetry $[\mathrm{O}(2) \otimes \mathrm{O}(N-1)] \oplus \mathrm{O}(2)$. For the discussion it is useful to introduce new couplings

$$
\begin{aligned}
& g_{1}=u_{0}+w_{0}, \\
& g_{2}=u_{0}+y_{0}, \\
& g_{3}=2 u_{0}+2 w_{0}-z_{0} .
\end{aligned}
$$

In terms of $g_{1}, g_{2}$, and $g_{3}$, the stability conditions for the quartic potential are particularly simple. We have $g_{1}>0, g_{2}>0, g_{3}>0$, and

$$
\begin{aligned}
u_{0}>-\sqrt{g_{2} g_{3} / 2} & \text { for } 0<g_{3}<2 g_{1}, \\
u_{0}>-\sqrt{g_{1} g_{2}} & \text { for } g_{3}>2 g_{1} .
\end{aligned}
$$

In order to solve the mean-field equations it is useful to use the symmetry in order to obtain a simple parametrization of the fields. Using the $\mathrm{O}(N-1)$ invariance for $\phi_{a i}$ and the $\mathrm{O}(2)$ invariance for $\psi$ we can write

$$
\phi_{a 1}=(a, 0, \ldots), \quad \phi_{a 2}=(b, c, 0 \ldots), \quad \psi_{i}=(d, 0) .
$$

Note that we have not used the additional $\mathrm{O}(2)$ symmetry transformations applied to the $\phi$ fields. If $a \neq 0$ and $b^{2}+c^{2} \neq 0$, we can perform $\mathrm{O}(2) \otimes \mathrm{O}(N-1)$ transformations to set $a^{2}=b^{2}+c^{2}$.

The mean-field equations are easily solved and we obtain six classes of solutions (we report one representative for each class; other solutions in each class are obtained by applying the symmetry transformations):

(a) $a=b=c=d=0$, with energy $H=0$. This is a minimum only if $r_{1}>0$ and $r_{2}>0$.

(b) $\phi_{1}^{2}=-6 r_{1} / g_{1}, \phi_{2}=0, \psi^{2}=0$, with energy $H=-\frac{3}{2} r_{1}^{2} / g_{1}$.

(c) $a^{2}=c^{2}=-6 r_{1} / g_{3}, b=d=0$, with energy $H=-3 r_{1}^{2} / g_{3}$.

(d) $\psi^{2}=-6 r_{2} / g_{2}, a=b=c=0$, with energy $H=-\frac{3}{2} r_{2}^{2} / g_{2}$.

(e) $a^{2}=-6\left(g_{2} r_{1}-r_{2} u_{0}\right) / D, d^{2}=-6\left(g_{1} r_{2}-r_{1} u_{0}\right) / D, b^{2}=c^{2}=0$, with $H=-\frac{3}{2}\left(g_{2} r_{1}^{2}+\right.$ $\left.g_{1} r_{2}^{2}-2 r_{1} r_{2} u_{0}\right) / D$, with $D \equiv g_{1} g_{2}-u_{0}^{2}$. 
(f) $a^{2}=c^{2}=-6\left(g_{2} r_{1}-r_{2} u_{0}\right) / D_{2}, d^{2}=-6\left(g_{3} r_{2}-2 r_{1} u_{0}\right) / D_{2}$, with $H=-\frac{3}{2}\left(2 g_{2} r_{1}^{2}+g_{3} r_{2}^{2}-\right.$ $\left.4 r_{1} r_{2} u_{0}\right) / D_{2}$, with $D_{2} \equiv g_{2} g_{3}-2 u_{0}^{2}$,

where $r_{1}=r_{\phi}$ and $r_{2}=r_{\psi}$. In order to determine the phase diagram, for each value of the couplings and of the ratio $r_{1} / r_{2}$, we must determine which solution has the lowest energy. The results are the following:

(1) $0<g_{3}<2 g_{1}$ and $u_{0}^{2}<g_{3} g_{2} / 2$. The critical point is tetracritical. For $r_{1}>0$ and $r_{2}>0$ the system is paramagnetic. Then, proceeding anticlockwise in the plane $\left(r_{1}, r_{2}\right)$ we find: a transition line for $r_{1}=0$ and $r_{2}>0$; phase (c); a transition line for $r_{2}=2 u_{0} r_{1} / g_{3}$ and $r_{1}<0$; phase (f); a transition line for $r_{2}=g_{2} r_{1} / u_{0}$ and $r_{2}<0$; phase (d); a transition line for $r_{2}=0$ and $r_{1}>0$. All transitions are continuous. In the presence of fluctuations transitions $(\mathrm{a}) /(\mathrm{d})$ and $(\mathrm{c}) /(\mathrm{f})$ belong to the XY universality class, while transitions $(\mathrm{a}) /(\mathrm{c})$ and $(\mathrm{d}) /(\mathrm{f})$ are chiral transitions, i.e. correspond to the symmetry breaking $\mathrm{O}(2) \otimes \mathrm{O}(N-1) \rightarrow \mathrm{O}(2) \otimes \mathrm{O}(N-3)$.

(2) $0<g_{3}<2 g_{1}$ and $u_{0}^{2}>g_{3} g_{2} / 2$. The critical point is bicritical. For $r_{1}>0$ and $r_{2}>0$ the system is paramagnetic. Then, proceeding anticlockwise in the plane $\left(r_{1}, r_{2}\right)$ we find: a transition line for $r_{1}=0$ and $r_{2}>0$; phase (c); a transition line for $r_{2}=r_{1} \sqrt{2 g_{2} / g_{3}}$ and $r_{1}<0$; phase $(\mathrm{d})$; a transition line for $r_{2}=0$ and $r_{1}>0$. Transitions $(\mathrm{a}) /(\mathrm{c})$ and $(\mathrm{a}) /(\mathrm{d})$ are continuous: transition $(\mathrm{a}) /(\mathrm{c})$ is a chiral one while transition $(\mathrm{a}) /(\mathrm{d})$ belongs to the XY universality class. Transition $(c) /(d)$ is of first order.

(3) $g_{3}>2 g_{1}$ and $u_{0}^{2}<g_{1} g_{2}$. The critical point is tetracritical. For $r_{1}>0$ and $r_{2}>0$ the system is paramagnetic. Then, proceeding anticlockwise in the plane $\left(r_{1}, r_{2}\right)$ we find: a transition line for $r_{1}=0$ and $r_{2}>0$; phase (b); a transition line for $r_{2}=u_{0} r_{1} / g_{1}$ and $r_{1}<0$; phase (e); a transition line for $r_{2}=g_{2} r_{1} / u_{0}$ and $r_{2}<0$; phase $(\mathrm{d})$; a transition line for $r_{2}=0$ and $r_{1}>0$. All transitions are continuous. In the presence of fluctuations transitions (a)/(d) and (b)/(e) belong to the XY universality class, while transitions $(\mathrm{a}) /(\mathrm{b})$ and $(\mathrm{d}) /(\mathrm{e})$ are collinear transitions, i.e. correspond to the symmetry breaking $\mathrm{O}(2) \otimes \mathrm{O}(N-1) \rightarrow \mathbb{Z}_{2} \otimes \mathrm{O}(N-2)$.

(4) $g_{3}>2 g_{1}$ and $u_{0}^{2}>g_{1} g_{2}$. The critical point is bicritical. For $r_{1}>0$ and $r_{2}>0$ the system is paramagnetic. Then, proceeding anticlockwise in the plane $\left(r_{1}, r_{2}\right)$ we find: a transition line for $r_{1}=0$ and $r_{2}>0$; phase (b); a transition line for $r_{2}=r_{1} \sqrt{g_{2} / g_{1}}$ and $r_{1}<0$; phase (d); a transition line for $r_{2}=0$ and $r_{1}>0$. Transitions (a)/(b) and $(\mathrm{a}) /(\mathrm{d})$ are continuous: transition $(\mathrm{a}) /(\mathrm{b})$ is a collinear one while transition $(\mathrm{a}) /(\mathrm{d})$ belongs to the XY universality class. Transition (b)/(d) is of first order.

\section{Model with $v_{0}>0$}

We shall now focus on the case $v_{0} \neq 0$. We shall only consider the case $v_{0}>0$, since the case $v_{0}<0$ can be recovered by using the symmetry (3.2). As before, we introduce new couplings 


$$
\begin{aligned}
& g_{1}=u_{0}+w_{0}, \\
& g_{2}=u_{0}+y_{0}, \\
& g_{3}=2 u_{0}+2 w_{0}-v_{0}-z_{0},
\end{aligned}
$$

that are all required to be positive by the stability of the quartic potential and are invariant under the symmetry (3.2). We also found additional necessary stability conditions. First, we must have

$$
\begin{aligned}
& u_{0}>v_{0}-\sqrt{g_{1} g_{2}}, \\
& u_{0}>-\sqrt{g_{1} g_{2}},
\end{aligned}
$$

where only the first condition is relevant for $v_{0}>0$. In order to write down the second condition let us define

$$
\begin{aligned}
R_{1} \equiv & \frac{1}{2} g_{2} g_{3}-\frac{1}{4} \frac{g_{3} v_{0}^{2}}{2 g_{1}-g_{3}}, \\
R_{2} \equiv & \frac{v_{0}^{2}}{2\left(2 g_{1}-g_{3}\right)}, \\
R_{3} \equiv & \left(2 g_{1} g_{2}-g_{2} g_{3}-2 g_{1} u_{0}+g_{3} u_{0}+g_{1} v_{0}-u_{0} v_{0}\right) \\
& \times\left(2 g_{1} g_{2}-g_{2} g_{3}-2 g_{1} u_{0}+g_{3} u_{0}+g_{1} v_{0}-g_{3} v_{0}+u_{0} v_{0}-v_{0}^{2}\right),
\end{aligned}
$$

and the domain $\Omega$ in the coupling space

$$
\Omega=\left\{\left(u_{0}, v_{0}, g_{1}, g_{2}, g_{3}\right): g_{3}<2 g_{1}, g_{2}>R_{2}, R_{3}>0\right\} .
$$

If $\left(u_{0}, v_{0}, g_{1}, g_{2}, g_{3}\right) \in \Omega$, then the couplings must satisfy

$$
u_{0}>\frac{v_{0}}{2}-\sqrt{R_{1}},
$$

We have not been able to prove that these conditions are sufficient for the stability of the quartic potential. However, since they go over to the stability conditions for $v_{0}=0$ (in this case we have proved they are sufficient), we believe they are enough for the stability of the quartic potential.

We parametrize the $\phi_{a i}$ fields as before, while for $\psi_{i}$ we must keep both components, i.e. we set $\psi_{i}=(d, e)$, because of the reduced symmetry of the model. Setting as before $r_{1}=r_{\phi}$ and $r_{2}=r_{\psi}$, the mean-field equations are

$$
\begin{aligned}
& a r_{1}+\frac{a}{6}\left[u_{0}\left(\phi^{2}+\psi^{2}\right)-v_{0}\left(c^{2}+e^{2}\right)+w_{0} \phi^{2}-z_{0} c^{2}\right]+\frac{1}{6} v_{0} b d e=0, \\
& b r_{1}+\frac{b}{6}\left[u_{0}\left(\phi^{2}+\psi^{2}\right)-v_{0} d^{2}+w_{0} \phi^{2}\right]+\frac{1}{6} v_{0} a d e=0, \\
& c r_{1}+\frac{c}{6}\left[u_{0}\left(\phi^{2}+\psi^{2}\right)-v_{0}\left(a^{2}+d^{2}\right)+w_{0} \phi^{2}-z_{0} a^{2}\right]=0, \\
& d r_{2}+\frac{d}{6}\left[u_{0}\left(\phi^{2}+\psi^{2}\right)-v_{0}\left(b^{2}+c^{2}\right)+y_{0} \psi^{2}\right]+\frac{1}{6} v_{0} a b e=0, \\
& e r_{2}+\frac{e}{6}\left[u_{0}\left(\phi^{2}+\psi^{2}\right)-v_{0} a^{2}+y_{0} \psi^{2}\right]+\frac{1}{6} v_{0} a b d=0 .
\end{aligned}
$$

The solutions are: 
(a) $a=b=c=d=e=0$, with energy $H=0$.

(b) $\phi_{1}^{2}=-6 r_{1} / g_{1}, \phi_{2}=0, \psi^{2}=0$, with energy $H=-\frac{3}{2} r_{1}^{2} / g_{1}$.

(c) $a^{2}=c^{2}=-6 r_{1} / g_{3}, b=d=e=0$, with energy $H=-3 r_{1}^{2} / g_{3}$.

(d) $\psi^{2}=-6 r_{2} / g_{2}, a=b=c=0$, with energy $H=-\frac{3}{2} r_{2}^{2} / g_{2}$.

(e1) $b^{2}=-6\left(g_{2} r_{1}-r_{2} u_{0}\right) / D, e^{2}=-6\left(g_{1} r_{2}-r_{1} u_{0}\right) / D, a^{2}=c^{2}=d^{2}=0$, with $H=$ $-\frac{3}{2}\left(g_{2} r_{1}^{2}+g_{1} r_{2}^{2}-2 r_{1} r_{2} u_{0}\right) / D$, with $D \equiv g_{1} g_{2}-u_{0}^{2}$.

$(\mathrm{e} 2) b^{2}=-6\left(g_{2} r_{1}-r_{2}\left(u_{0}-v_{0}\right)\right) / D_{3}, d^{2}=-6\left(g_{1} r_{2}-r_{1}\left(u_{0}-v_{0}\right)\right) / D_{3}, a^{2}=c^{2}=e^{2}=0$, with $H=-\frac{3}{2}\left(g_{2} r_{1}^{2}+g_{1} r_{2}^{2}-2 r_{1} r_{2}\left(u_{0}-v_{0}\right)\right) / D_{3}$, with $D_{3} \equiv g_{1} g_{2}-\left(u_{0}-v_{0}\right)^{2}$.

(f) This solution is too long to be reported. Both vectors are nonvanishing, and we can take $a^{2}=b^{2}+c^{2}, b^{2}=O\left(v_{0}^{2}\right)$. Moreover, $d^{2}=e^{2}$ and $d e /(a b)=\left(g_{3}-2 g_{1}\right) / v_{0}$.

The solutions follow the labelling used for $v_{0}=0$. Note that for $v_{0}>0$ there are two different solutions corresponding the solution (e) found before. The derivation of these solutions is straightforward, except for case ( $\mathrm{f}$ ). To derive (f), assume that $a, b, c, d$, and $e$ are nonvanishing. Then, Eqs. (A9) and (A10) imply

$$
d e=a b \frac{g_{3}-2 g_{1}}{v_{0}} .
$$

Substitute this relation in Eqs. (A8) and (A9). Analogously, one can use this relation to express $a b$ in terms of de in Eqs. (A11) and (A12). The five mean-field equations become linear in $a^{2}, b^{2}, c^{2}, d^{2}$, and $e^{2}$. However, only three of them are independent. To completely solve the problem, we consider Eqs. (A8), (A9), and (A11) that are independent, the equation $a^{2}=b^{2}+c^{2}$ that fixes the $\mathrm{O}(2)$ invariance, and the relation between $d^{2} e^{2}$ and $a^{2} b^{2}$ that follows from Eq. (A13). This system of equations has a unique solution for $a^{2}, b^{2}, c^{2}, d^{2}$, and $e^{2}$.

The analysis of the phase diagram of this model is extremely complex, mainly due to the cumbersome expressions for solution (f). We have used analytic and numerical methods to sort out the different possibilities. We find:

(a) We have a pentacritical point for $2 g_{1}-g_{3}>0, g_{2}>R_{2}$, and $v_{0} / 2-\sqrt{R_{1}}<u_{0}<$ $v_{0} / 2+\sqrt{R_{1}}$. For $r_{1}>0$ and $r_{2}>0$ the system is paramagnetic. Then, proceeding anticlockwise in the plane $\left(r_{1}, r_{2}\right)$ we find: a transition line for $r_{1}=0$ and $r_{2}>0$; phase (c); a transition line for $r_{1}=g_{3} r_{2} /\left(2 u_{0}-v_{0}\right)$ and $r_{1}<0$; phase (f); a transition line; phase (e2); a transition line for $r_{1}=\left(u_{0}-v_{0}\right) r_{2} / g_{2}$ and $r_{2}<0$; phase $(\mathrm{d})$; a transition line for $r_{2}=0$ and $r_{1}>0$. All transitions are continuous except that between phases (f) and (e2) that is of first order. In the presence of fluctuations transitions (a)/(d) and $(\mathrm{c}) /(\mathrm{f})$ belong to the XY universality class, transition $(\mathrm{d}) /(\mathrm{e} 2)$ belongs to the $\mathrm{O}(N-1)$ vector universality class, while transition $(\mathrm{a}) /(\mathrm{c})$ is a chiral transition, i.e. it corresponds to the symmetry breaking $\mathrm{O}(2) \otimes \mathrm{O}(N-1) \rightarrow \mathrm{O}(2) \otimes \mathrm{O}(N-3)$. For $v_{0} \rightarrow 0$ the width of phase (e2) goes to zero and we obtain case (1) considered in the previous Section. 
(b) We have a tetracritical point in three cases: $2 g_{1}-g_{3}>0, g_{2}>R_{2}, u_{0}<v_{0} / 2-\sqrt{R_{1}}$ up to the stability boundary; $2 g_{1}-g_{3}>0, g_{2}>R_{2}, v_{0} / 2+\sqrt{R_{1}}<u_{0}<v_{0}+\sqrt{g_{2} g_{3} / 2}$; $2 g_{1}-g_{3}>0,0<g_{2}<R_{2}, u_{0}<v_{0}+\sqrt{g_{2} g_{3} / 2}$ up to the stability boundary. For $r_{1}>0$ and $r_{2}>0$ the system is paramagnetic. Then, proceeding anticlockwise in the plane $\left(r_{1}, r_{2}\right)$ we find: a transition line for $r_{1}=0$ and $r_{2}>0$; phase (c); a transition line with $r_{1}<0$; phase (e2); a transition line with $r_{1}=\left(u_{0}-v_{0}\right) r_{2} / g_{2}$ and $r_{2}<0$; phase (d); a transition line for $r_{2}=0$ and $r_{1}>0$. Transitions (a)/(c), (e2)/(d), (d)/(a) are continuous, while transition $(\mathrm{c}) /(\mathrm{e} 2)$ is of first order. In the presence of fluctuations transition $(\mathrm{a}) /(\mathrm{d})$ belongs to the XY universality class, transition $(\mathrm{d}) /(\mathrm{e} 2)$ belongs to the $\mathrm{O}(N-1)$ vector universality class, while transition $(\mathrm{a}) /(\mathrm{c})$ is a chiral transition, i.e. it corresponds to the symmetry breaking $\mathrm{O}(2) \otimes \mathrm{O}(N-1) \rightarrow \mathrm{O}(2) \otimes \mathrm{O}(N-3)$. Such a case does not exist for $v_{0}=0$.

(c) We have a bicritical point for $2 g_{1}-g_{3}>0$ and $u_{0}>v_{0}+\sqrt{g_{2} g_{3} / 2}$. For $r_{1}>0$ and $r_{2}>0$ the system is paramagnetic. Then, proceeding anticlockwise in the plane $\left(r_{1}, r_{2}\right)$ we find: a transition line for $r_{1}=0$ and $r_{2}>0$; phase (c); a transition line for $r_{1}=r_{2}\left(g_{3} / 2 g_{2}\right)^{1 / 2}$; phase (d); a transition line for $r_{2}=0$ and $r_{1}>0$. Transition (c) /(d) is of first order, transition (a)/(c) is a chiral transition, while transition $(\mathrm{a}) /(\mathrm{d})$ belongs to the XY universality class.

(d) We have a tetracritical point for $2 g_{1}-g_{3}<0$ and $v_{0}-\sqrt{g_{1} g_{2}}<u_{0}<v_{0}+\sqrt{g_{1} g_{2}}$. For $r_{1}>0$ and $r_{2}>0$ the system is paramagnetic. Then, proceeding anticlockwise in the plane $\left(r_{1}, r_{2}\right)$ we find: a transition line for $r_{1}=0$ and $r_{2}>0$; phase (b); a transition line for $r_{1}=g_{1} r_{2} /\left(u_{0}-v_{0}\right)$ and $r_{1}<0$; phase $(\mathrm{e} 2) ; r_{1}=\left(u_{0}-v_{0}\right) r_{2} / g_{2}$ and $r_{2}<0$; phase (d); a transition line for $r_{2}=0$ and $r_{1}>0$. All transitions are continuous. Transition $(\mathrm{a}) /(\mathrm{b})$ is a collinear transition with $v_{0}<0$, i.e. it corresponds to the symmetry breaking $\mathrm{O}(2) \otimes \mathrm{O}(N-1) \rightarrow \mathbb{Z}_{2} \otimes \mathrm{O}(N-2)$, transitions (b)/(e2) and $(\mathrm{a}) /(\mathrm{d})$ are XY transitions, while transition $(\mathrm{d}) /(\mathrm{e} 2)$ belongs to the $\mathrm{O}(N-1)$ vector universality class.

(e) We have a bicritical point for $2 g_{1}-g_{3}<0$ and $u_{0}>v_{0}+\sqrt{g_{1} g_{2}}$. For $r_{1}>0$ and $r_{2}>0$ the system is paramagnetic. Then, proceeding anticlockwise in the plane $\left(r_{1}, r_{2}\right)$ we find: a transition line for $r_{1}=0$ and $r_{2}>0$; phase (b); a transition line for $r_{1}=r_{2}\left(g_{1} / g_{2}\right)^{1 / 2}$; phase $(\mathrm{d})$; a transition line for $r_{2}=0$ and $r_{1}>0$. Transition (a)/(b) is a collinear transition with $v_{0}<0$, transition $(\mathrm{b}) /(\mathrm{d})$ is of first order, and transition (a)/(d) belongs to the XY universality class.

\section{APPENDIX B: CLASSIFICATION OF THE PERTURBATIONS OF AN $\mathbf{O}(M) \otimes \mathbf{O}(N)$ THEORY}

We consider an $\mathrm{O}(M) \otimes \mathrm{O}(N)$-symmetric theory with $M, N \geq 2$ and the Hamiltonian

$$
\mathcal{H}_{M N}=\int d^{d} x\left\{\frac{1}{2} \sum_{a i}\left[\left(\partial_{\mu} \Phi_{a i}\right)^{2}+r \Phi_{a i}^{2}\right]\right.
$$




$$
\left.+\frac{1}{4 !} u_{0}\left(\sum_{a i} \Phi_{a i}^{2}\right)^{2}+\frac{1}{4 !} v_{0} \sum_{a, b}\left[\left(\sum_{i} \Phi_{a i} \Phi_{b i}\right)^{2}-\left(\sum_{i} \Phi_{a}^{2}\right)\left(\sum_{i} \Phi_{b i}\right)^{2}\right]\right\}
$$

where $\Phi_{a i}(a=1, \ldots N$ and $i=1, \ldots M)$ are $N \times M$ matrix variables. We wish now to classify the quadratic and quartic operators that break the $\mathrm{O}(N)$ symmetry but preserve the $\mathrm{O}(M)$ symmetry. The classification of all the quadratic operators that break both symmetries is presented in App. C.

At the quadratic level, there is only one operator, the spin-2 operator

$$
\mathcal{O}_{a b}^{(2,2)}=\Phi_{a} \cdot \Phi_{b}-\frac{1}{N} \delta_{a b} \Phi^{2}
$$

where (as in all this Section) the scalar product is in the $\mathrm{O}(M)$ space and $\Phi^{2}=\sum_{a} \Phi_{a} \cdot \Phi_{a}$.

At the quartic level, there are three operators, one spin-4 operator, two spin-2 operators and one operator associated with a nontrivial $\mathrm{O}(N)$ representation. The spin-4 operator is given by

$$
\begin{aligned}
\mathcal{O}_{a b c d}^{(4,4)}= & \left(\Phi_{a} \cdot \Phi_{b}\right)\left(\Phi_{c} \cdot \Phi_{d}\right)+2 \text { perm. } \\
& -\frac{1}{N+4} \Phi^{2}\left(\delta_{a b}\left(\Phi_{c} \cdot \Phi_{d}\right)+5 \text { perm. }\right) \\
& -\frac{2}{N+4}\left(\delta_{a b} \sum_{e}\left(\Phi_{e} \cdot \Phi_{c}\right)\left(\Phi_{e} \cdot \Phi_{d}\right)+5 \text { perm. }\right) \\
& +\frac{1}{(N+2)(N+4)}\left(\Phi^{2}\right)^{2}\left(\delta_{a b} \delta_{c d}+2 \text { perm. }\right) \\
& +\frac{2}{(N+2)(N+4)}\left[\sum_{e f}\left(\Phi_{e} \cdot \Phi_{f}\right)^{2}\right]\left(\delta_{a b} \delta_{c d}+2 \text { perm. }\right)
\end{aligned}
$$

where the permutations are such to make $\mathcal{O}_{a b c d}^{(4,4)}$ symmetric and traceless. The spin-2 operators are given by

$$
\begin{aligned}
& \mathcal{O}_{a b}^{(4,2,1)}=\Phi^{2} \mathcal{O}_{a b}^{(2,2)} \\
& \mathcal{O}_{a b}^{(4,2,2)}=\sum_{e}\left(\Phi_{a} \cdot \Phi_{e}\right)\left(\Phi_{b} \cdot \Phi_{e}\right)-\frac{1}{N} \delta_{a b}\left[\sum_{e f}\left(\Phi_{e} \cdot \Phi_{f}\right)^{2}\right] .
\end{aligned}
$$

They are always independent except for $N=2$. In this case $\mathcal{O}_{a b}^{(4,2,1)}=\mathcal{O}_{a b}^{(4,2,2)}$.

The remaining operator is

$$
\begin{aligned}
\mathcal{O}_{a b c d}^{(4, r)}= & \left(\Phi_{a} \cdot \Phi_{c}\right)\left(\Phi_{b} \cdot \Phi_{d}\right)-\left(\Phi_{a} \cdot \Phi_{d}\right)\left(\Phi_{b} \cdot \Phi_{c}\right) \\
& -\frac{1}{N(N-1)}\left(\delta_{a c} \delta_{b d}-\delta_{a d} \delta_{b c}\right)\left[\left(\Phi^{2}\right)^{2}-\sum_{e f}\left(\Phi_{e} \cdot \Phi_{f}\right)^{2}\right] \\
& -\frac{\delta_{a c}}{N-2}\left(\mathcal{O}_{b d}^{(4,2,1)}-\mathcal{O}_{b d}^{(4,2,2)}\right)-\frac{\delta^{b d}}{N-2}\left(\mathcal{O}_{a c}^{(4,2,1)}-\mathcal{O}_{a c}^{(4,2,2)}\right) \\
& +\frac{\delta^{a d}}{N-2}\left(\mathcal{O}_{b c}^{(4,2,1)}-\mathcal{O}_{b c}^{(4,2,2)}\right)+\frac{\delta^{b c}}{N-2}\left(\mathcal{O}_{a d}^{(4,2,1)}-\mathcal{O}_{a d}^{(4,2,2)}\right) .
\end{aligned}
$$


Such a quantity satisfies the properties $\mathcal{O}_{a b c d}^{(4, r)}=-\mathcal{O}_{b a c d}^{(4, r)}, \mathcal{O}_{a b c d}^{(4, r)}=-\mathcal{O}_{a b d c}^{(4, r)}, \mathcal{O}_{a b c d}^{(4, r)}=\mathcal{O}_{c d a b}^{(4, r)}$, $\sum_{c=1}^{N} \mathcal{O}_{\text {cacb }}^{(4, r)}=0$. It belongs to a nontrivial representation of the $\mathrm{O}(N)$ group, the one associated with the Young tableau that has the shape of a $2 \times 2$ box. (Note that in our terminology we call spin- $k$ representation the representation associated with the Young tableau that has the shape of a row of length $k$ ). This representation occurs only for $N \geq 4$. Indeed, this operator is not defined for $N=2$; for $N=3$ we have $\sum_{a b c d} \epsilon^{e a b} \epsilon^{f c d} \mathcal{O}_{a b c d}^{(4, r)}=0$, which shows that the operator does not occur. The operator $\mathcal{O}_{a b c d}^{(4, r)}$ does not mix with any other (quadratic or quartic) operator.

These operators control the symmetry breaking

$$
O(M) \otimes O(N) \rightarrow O(M) \otimes[O(k) \oplus O(N-k)] .
$$

The corresponding multicritical Hamiltonian is

$$
\mathcal{H}_{N, k}=\mathcal{H}_{M N}+m_{2} V^{(2,2)}+f_{1} V^{(4,4)}+f_{2} V^{(4,2,1)}+f_{3} V^{(4,2,2)}+f_{4} V^{(4, r)},
$$

where $V^{(2,2)}=\sum_{a=1}^{k} \mathcal{O}_{a a}^{(2,2)}, V^{(4,4)}=\sum_{a=1}^{k} \sum_{b=k+1, n} \mathcal{O}_{a a b b}^{(4,4)}, V^{(4,2,1)}=\sum_{a=1}^{k} \mathcal{O}_{a a}^{(4,2,1)}, V^{(4,2,2)}=$ $\sum_{a=1}^{k} \mathcal{O}_{a a}^{(4,2,2)}, V^{(4, r)}=\sum_{a b=1}^{k} \mathcal{O}_{a b a b}^{(4, r)}$. For $k=1$ or $k=N-1, V^{(4, r)}$ vanishes. Indeed, if $k=1$ $V^{(4, r)}=\mathcal{O}_{1111}^{(4, r)}$ that vanishes because of the antisymmetry of the indices. For $k=N-1$, using the fact that $\mathcal{O}_{a b c d}^{(4, r)}$ is traceless, we have $V^{(4, r)}=-\sum_{a=1}^{k} \mathcal{O}_{a N a N}^{(4, r)}=\mathcal{O}_{N N N N}^{(4, r)}=0$.

Explicitly, if $\Phi_{a m} \rightarrow\left(\phi_{A m}, \psi_{\alpha m}\right)$ with $A=1, \ldots k, \alpha=1, \ldots N-k, m=1, \ldots M$, we have

$$
\begin{aligned}
V^{(2,2)}= & \frac{N-k}{N} \phi^{2}-\frac{k}{N} \psi^{2}, \\
V^{(4,4)}= & \frac{1}{(N+2)(N+4)}\left\{-(N-k)(N-k+2)\left[\left(\phi^{2}\right)^{2}+2 \sum_{A B}\left(\phi_{A} \cdot \phi_{B}\right)^{2}\right]\right. \\
& \left.-k(k+2)\left[\left(\psi^{2}\right)^{2}+2 \sum_{\alpha \beta}\left(\psi_{\alpha} \cdot \psi_{\beta}\right)^{2}\right]+2(k+2)(N-k+2)\left[\phi^{2} \psi^{2}+2 \sum_{A \alpha}\left(\phi_{A} \cdot \psi_{\alpha}\right)^{2}\right]\right\}, \\
V^{(4,2,1)}= & \left(\phi^{2}+\psi^{2}\right) V^{(2,2)}, \\
V^{(4,2,2)}= & \frac{N-k}{N} \sum_{A B}\left(\phi_{A} \cdot \phi_{B}\right)^{2}+\frac{N-2 k}{N} \sum_{A \alpha}\left(\phi_{A} \cdot \psi_{\alpha}\right)^{2}-\frac{k}{N} \sum_{\alpha \beta}\left(\psi_{\alpha} \cdot \psi_{\beta}\right)^{2}, \\
V^{(4, r)=} & -\frac{1}{(N-1)(N-2)}\left\{-(N-k)(N-k-1)\left[\left(\phi^{2}\right)^{2}-\sum_{A B}\left(\phi_{A} \cdot \phi_{B}\right)^{2}\right]\right. \\
& \left.\left.-k(k-1)\left[\left(\psi^{2}\right)^{2}-\sum_{\alpha \beta}\left(\psi_{\alpha} \cdot \psi_{\beta}\right)^{2}\right]+2(k-1)(N-k-1)\left[\phi^{2} \psi^{2}-\sum_{A \alpha}\left(\phi_{A} \cdot \psi_{\alpha}\right)^{2}\right]\right\} .(\mathrm{B} 9)\right\}
\end{aligned}
$$

It is trivial to check that $V^{(4, r)}$ vanishes for $k=1$ or $k=N-1$.

In conclusion, at the quartic level: for $N=2$ there are two breaking operators, $V^{(4,2,1)}$ and $V^{(4,4)}$; for $N=3$ and for $N \geq 4, k=1$ or $k=N-1$, there are three breaking operators, 
$V^{(4,2,1)}, V^{(4,2,2)}$, and $V^{(4,4)}$; for $N \geq 4$ and $2 \leq k \leq N-2$, all four operators should be considered.

These operators are also relevant in other cases. If we consider the breaking

$$
O(M) \otimes O(N) \rightarrow O(M) \otimes C_{N}
$$

where $C_{N}$ is the cubic group in an $N$-dimensional space, we obtain the Hamiltonian

$$
\mathcal{H}_{M N}+f \sum_{a=1}^{N} \mathcal{O}_{\text {aaaa }}^{(4,4)}
$$

For $N=4$ we may consider the breaking

$$
O(M) \otimes O(4) \rightarrow O(M) \otimes S O(4)
$$

The corresponding Hamiltonian is

$$
\mathcal{H}_{M N}+f \sum_{a b c d=1}^{N} \epsilon^{a b c d} \mathcal{O}_{a b c d}^{(4, r)}
$$

Note, finally, that reduction to smaller symmetry groups does not require the consideration of additional operators, although there may be additional terms in the Hamiltonian. For instance, the breaking

$$
O(M) \otimes O(N) \rightarrow O(M) \otimes\left(C_{k} \oplus C_{N-k}\right)
$$

is obtained by considering

$$
\mathcal{H}_{N, k}+f_{5} \sum_{a=1}^{k} \mathcal{O}_{\text {aaaa }}^{(4,4)}+f_{6} \sum_{b=k+1}^{N} \mathcal{O}_{b b b b}^{(4,4)}
$$

The operators proportional to $f_{1}, f_{5}$, and $f_{6}$ are of course degenerate at the $\mathrm{O}(M) \otimes \mathrm{O}(N)$ FP.

\section{APPENDIX C: RG DIMENSIONS OF THE QUADRATIC PERTURBATIONS AT THE $\mathbf{O}(2) \otimes O(N)$ FIXED POINTS}

In this appendix we consider the three-dimensional $\mathrm{O}(2) \otimes \mathrm{O}(N)$ invariant theory, cfr. Eq. (B1) with $M=2$, and compute the RG dimensions of all quadratic operators breaking the $\mathrm{O}(2) \otimes \mathrm{O}(N)$ symmetry at the $\mathrm{O}(2) \otimes \mathrm{O}(N)$ FPs.

For generic $M$, the quadratic operators breaking the $\mathrm{O}(M) \otimes \mathrm{O}(N)$ symmetry are explicitly given by 


$$
\begin{aligned}
O_{a i b j}^{(1)} & =\Phi_{a i} \Phi_{b j}-\Phi_{a j} \Phi_{b i} \\
O_{a i b j}^{(2)} & =\frac{1}{2}\left(\Phi_{a i} \Phi_{b j}+\Phi_{a j} \Phi_{b i}\right)-\frac{1}{N} \delta_{a b} \sum_{c} \Phi_{c i} \Phi_{c j}-\frac{1}{M} \delta_{i j} \sum_{k} \Phi_{a k} \Phi_{b k}+\frac{1}{M N} \delta_{a b} \delta_{i j} \sum_{c k} \Phi_{c k} \Phi_{c k} \\
O_{a b}^{(3)} & =\sum_{k} \Phi_{a k} \Phi_{b k}-\frac{1}{N} \delta_{a b} \sum_{c k} \Phi_{c k} \Phi_{c k} \\
O_{i j}^{(4)} & =\sum_{c} \Phi_{c i} \Phi_{c j}-\frac{1}{M} \delta_{i j} \sum_{c k} \Phi_{c k} \Phi_{c k}
\end{aligned}
$$

where $\Phi_{a i}$ is a real field with $a=1, \ldots, N$ and $i=1, \ldots, M$. These operators have a simple group-theory intepretation, that allows us to check that the list is exhaustive. The operator $O_{a i b j}^{(1)}$ transforms as a spin-1 operator under both $\mathrm{O}(N)$ and $\mathrm{O}(M), O_{\text {aibj }}^{(2)}$ transforms as a spin-2 operator under both groups, while $O_{a b}^{(3)}$ and $O_{i j}^{(4)}$ transform as a scalar under one group and as a spin- 2 operator under the second group. For $M=2$ these operators correspond to those reported in Ref. [14].

For $M=2$, we computed the RG dimensions $y_{i}$ of the above-reported quadratic operators to six loops in the MZM scheme and to five loops in the $3 d-\overline{\mathrm{MS}}$ scheme. We used a symbolic program to generate diagrams and group factors and the compilations of Feynman integrals of Refs. [39,25]. We do not report the series that are available on request. The results of the analyses, using the resummation methods outlined in Refs. [40,19,20], are reported in Table III for several values of $N$. In the case $N=6$ we do not report results for the chiral $\mathrm{FP}$ in the MZM scheme, since in this scheme there is little evidence for the existence of a $\mathrm{FP}$. The RG dimensions of the operator $O^{(1)}$ related to the chiral degrees of freedom at the chiral FP have already been computed by exploiting the same FT methods in Refs. [44,20]; we report them here for the sake of completeness. We also mention that these exponents have been computed to order $1 / N$ in Ref. [14], while Ref. [45] reports a $1 / N^{2}$ calculation of the RG dimension of $O^{(1)}$.

For $N=2$ the RG dimensions at the collinear FP can be related to the RG dimensions of operators in the XY model. Indeed, the $\mathrm{O}(2) \otimes \mathrm{O}(2)$ collinear FP is equivalent to an XY FP. The mapping is the following. One defines two fields $a_{i}$ and $b_{i}, i=1,2$, and considers $[14]$

$$
\begin{aligned}
& \phi_{11}=\left(a_{1}-b_{2}\right) / \sqrt{2}, \\
& \phi_{22}=\left(a_{1}+b_{2}\right) / \sqrt{2}, \\
& \phi_{12}=\left(b_{1}-a_{2}\right) / \sqrt{2}, \\
& \phi_{21}=\left(b_{1}+a_{2}\right) / \sqrt{2} .
\end{aligned}
$$

At the collinear FP, fields $a$ and $b$ represent two independent XY fields. Using this mapping it is easy to show that: $O_{a i b j}^{(1)} \sim a^{2}+b^{2} ; O_{a i b j}^{(2)}$ is the sum of $a_{1}^{2}-a_{2}^{2}, b_{1}^{2}-b_{2}^{2}, a_{1} a_{2}$, and $b_{1} b_{2}$; $O_{a b}^{(3)}$ (or $O_{i j}^{(4)}$ ) is the sum of terms of the form $a_{i} b_{j}$. Thus, $y_{1}=y_{t, \mathrm{XY}}=1 / \nu_{\mathrm{XY}}, y_{2}=y_{T, \mathrm{XY}}$, where $y_{T, \mathrm{XY}}$ is the RG dimension of the spin-2 quadratic operator in the XY model, and $y_{3}=y_{4}=2 y_{h, \mathrm{XY}}-3$, where $y_{h, \mathrm{XY}}=\left(5-\eta_{\mathrm{XY}}\right) / 2$ is the $\mathrm{RG}$ dimension of the field in the $\mathrm{XY}$ model. Note also that the scalar operator $\phi^{2}$ becomes $a^{2}+b^{2}$, as obviously expected. Estimates of $\nu_{\mathrm{XY}}, \eta_{\mathrm{XY}}$, and $y_{T, \mathrm{XY}}$ can be found in Refs. [38,22,18,42]. 
TABLE III. RG dimensions of the quadratic operators breaking the symmetry $O(2) \otimes O(N)$ at the chiral (ch) and collinear (cl) FPs. We report the estimates obtained by analyzing the $\overline{\mathrm{MS}}$ (five loops) and MZM (six loops) expansions. The errors include the spread of the considered approximants and the uncertainty on the location of the FP. The results for $N=2$ at the collinear FP have been obtained by using the mapping with the XY model and the numerical results reported in Refs. [38,18].

\begin{tabular}{|c|c|c|c|c|c|c|c|c|}
\hline \multirow[t]{2}{*}{$\mathrm{FP}, N$} & \multicolumn{2}{|c|}{$y_{1}$} & \multicolumn{2}{|c|}{$y_{2}$} & \multicolumn{2}{|c|}{$y_{3}$} & \multicolumn{2}{|c|}{$y_{4}$} \\
\hline & $\overline{\mathrm{MS}}$ & MZM & $\overline{\mathrm{MS}}$ & MZM & $\overline{\mathrm{MS}}$ & MZM & $\overline{\mathrm{MS}}$ & MZM \\
\hline$\overline{\mathrm{ch}, 2}$ & $2.37(15)$ & $2.54(12)$ & $2.00(15)$ & $2.07(7)$ & $1.34(15)$ & $1.25(4)$ & $1.34(15)$ & $1.25(4)$ \\
\hline $\operatorname{ch}, 3$ & $2.25(12)$ & $2.35(13)$ & $1.96(11)$ & $1.99(4)$ & $1.54(8)$ & $1.49(3)$ & $1.21(9)$ & $1.09(5)$ \\
\hline $\mathrm{ch}, 4$ & $2.17(10)$ & $2.29(8)$ & $1.94(10)$ & $2.04(20)$ & $1.65(3)$ & $1.64(5)$ & $1.17(8)$ & $1.06(7)$ \\
\hline $\operatorname{ch}, 5$ & $2.05(10)$ & $2.20(7)$ & $1.93(10)$ & $1.98(20)$ & $1.72(4)$ & $1.72(8)$ & $1.15(7)$ & $1.02(8)$ \\
\hline $\operatorname{ch}, 6$ & $2.03(7)$ & & $1.90(10)$ & & $1.76(4)$ & & $1.13(9)$ & \\
\hline $\mathrm{ch}, 8$ & $2.02(2)$ & $2.03(4)$ & $1.92(2)$ & $1.93(4)$ & $1.81(2)$ & $1.79(1)$ & $1.13(8)$ & $1.13(4)$ \\
\hline $\mathrm{ch}, 16$ & $2.001(5)$ & $2.00(1)$ & $1.948(5)$ & $1.95(1)$ & $1.897(7)$ & $1.885(5)$ & $1.08(2)$ & $1.07(1)$ \\
\hline $\operatorname{ch}, \infty$ & \multicolumn{2}{|c|}{2} & \multicolumn{2}{|c|}{2} & \multicolumn{2}{|r|}{2} & \multicolumn{2}{|r|}{1} \\
\hline $\mathrm{cl}, 2$ & \multicolumn{2}{|c|}{$y_{t, \mathrm{XY}}=1.489(6)$} & \multicolumn{2}{|c|}{$y_{T, \mathrm{XY}}=1.766(6)$} & \multicolumn{2}{|c|}{$2 y_{h, \mathrm{XY}}-3=1.9620(8)$} & \multicolumn{2}{|c|}{$2 y_{h, \mathrm{XY}}-3=1.9620(8)$} \\
\hline $\mathrm{cl}, 3$ & $1.2(1)$ & $1.15(10)$ & $1.75(5)$ & $1.75(10)$ & $2.0(1)$ & $2.0(2)$ & $2.1(2)$ & $2.05(15)$ \\
\hline $\mathrm{cl}, 4$ & $1.1(1)$ & $1.10(15)$ & $1.65(10)$ & $1.66(5)$ & $1.90(15)$ & $1.75(10)$ & $2.0(3)$ & $2.05(15)$ \\
\hline
\end{tabular}

\section{REFERENCES}

[1] M.F. Collins, O.A. Petrenko, Can. J. Phys. 75 (1997) 605.

[2] H. Kawamura, J. Phys.: Condens. Matter 10 (1998) 4707 [cond-mat/9805134].

[3] P.B. Johnson, J.A. Rayne, S.A. Friedberg, J. Appl. Phys. 50 (1979) 583.

[4] D. Beckmann, J. Wosnitza, H. von Löhneysen, Phys. Rev. Lett. 71 (1993) 2829.

[5] M. Enderle, F. Fortuna, M. Steiner, J. Phys.: Condens. Matter 6 (1994) L385.

[6] M. Enderle, R. Schneider, Y. Matsuoka, K. Kakurai, Physica B 234-36 (1997) 554.

[7] M. Poirier, A. Caillé, M.L. Plumer, Phys. Rev. B 41 (1990) 4869.

[8] H.A. Katori, T. Goto, Y. Ajiro, J. Phys. Soc. Jpn. 62 (1993) 743.

[9] T. Asano, Y. Ajiro, M. Mekata, H.A. Katori, T. Goto, Physica B 201 (1994) 75.

[10] M.L. Plumer, K. Hood, A. Caillé, Phys. Rev. Lett. 60 (1988) 45.

[11] M.L. Plumer, A. Caillé, Phys. Rev. B 41 (1990) 2543.

[12] H. Kawamura, A. Caillé, M.L. Plumer, Phys. Rev. B 41 (1990) 4416.

[13] A. Mailhot, M.L. Plumer, A. Caillé, Phys. Rev. B 48 (1993) 15835.

[14] H. Kawamura, Phys. Rev. B 38 (1988) 4916; erratum B 42 (1990) 2610.

[15] G.A. Baker, Phys. Rev. 126 (1962) 2071.

[16] J. Hubbard, Phys. Lett. 39A (1972) 365.

[17] J.M. Kosterlitz, D.R. Nelson, M.E. Fisher, Phys. Rev. B 13 (1976) 412.

[18] P. Calabrese, A. Pelissetto, E. Vicari, Phys. Rev. B 67 (2003) 054505 [condmat/0209580].

[19] A. Pelissetto, P. Rossi, E. Vicari, Phys. Rev. B 63 (2001) 140414(R) [cond-mat/0007389].

[20] P. Calabrese, P. Parruccini, A. Pelissetto, E. Vicari, cond-mat/0405667.

[21] M. Tissier, B. Delamotte, D. Mouhanna, Phys. Rev. Lett. 84 (2000) 5208 [condmat/0001350].

[22] A. Pelissetto, E. Vicari, Phys. Rept. 368 (2002) 549 [cond-mat/0012164].

[23] B. Delamotte, D. Mouhanna, M. Tissier, Phys. Rev. B 69 (2004) 134413 [condmat/0309101]. 
[24] R. Schloms, V. Dohm, Nucl. Phys. B 328 (1989) 639; Phys. Rev. B 42 (1990) 6142.

[25] H. Kleinert, V. Schulte-Frohlinde, Critical Properties of $\phi^{4}$-Theories (World Scientific, Singapore, 2001).

[26] G. Parisi, Cargèse Lectures (1973), J. Stat. Phys. 23 (1980) 49.

[27] S.N. Coppersmith, Phys. Rev. B 32 (1985) 1584.

[28] D. Blankschtein, M. Ma, A. Nihat Berker, G.S. Grest, C.M. Soukoulis, Phys. Rev. B 29 (1984) 5250 .

[29] A. Nihat Berker, G.S. Grest, C.M. Soukoulis, D. Blankschtein, M. Ma, J. Appl. Phys. 55 (1984) 2416.

[30] M.L. Plumer, A. Caillé, K. Hood, Phys. Rev. B 40 (1989) 4958.

[31] M. De Prato, A, Pelissetto, E. Vicari to appear in Phys. Rev. B 70 (2004) [condmat/0312362].

[32] P. Calabrese, P. Parruccini, A.I. Sokolov, Phys. Rev. B 66 (2002) 180403 [condmat/0205046]; B 68 (2003) 094415 [cond-mat/0304154].

[33] S.A. Antonenko, A.I. Sokolov, V.B. Varnashev, Phys. Lett. A 208 (1995) 161 [condmat/9803377].

[34] A. Pelissetto, P. Rossi, E. Vicari, Nucl. Phys. B 607 (2001) 605 [hep-th/0104024].

[35] P. Calabrese, P. Parruccini, Nucl. Phys. B 679 (2004) 568 [cond-mat/0308037].

[36] M. Kindermann, C. Wetterich, Phys. Rev. Lett. 86 (2001) 1034 [cond-mat/0008332].

[37] A. Aharony, Phys. Rev. Lett. 88 (2002) 059703 [cond-mat/0107585]; J. Stat. Phys. 110 (2003) 659 [cond-mat/0201576].

[38] M. Campostrini, M. Hasenbusch, A. Pelissetto, P. Rossi, E. Vicari, Phys. Rev. B 63 (2001) 214503 [cond-mat/0010360].

[39] B. G. Nickel, D. I. Meiron, G. A. Baker, Jr., "Compilation of 2-pt, 4-pt graphs for continuum spin models," Guelph University Report, 1977, unpublished.

[40] J.M. Carmona, A. Pelissetto, E. Vicari, Phys. Rev. B 61 (2000) 15136 [condmat/9912115].

[41] P. Calabrese, A. Pelissetto, E. Vicari, Phys. Rev. E 65 (2002) 046115 [condmat/0111160].

[42] P. Calabrese, A. Pelissetto, E. Vicari, in Frontiers in Superconductivity Research, edited by Barry P. Martins (Nova Science, Hauppauge, NY, 2004); cond-mat/0306273.

[43] H. Kadowaki, T. Inami, Y. Ajiro, K. Nakajima, Y. Endoh, J. Phys. Soc. Jpn. 60 (1991) 1708.

[44] A. Pelissetto, P. Rossi, E. Vicari, Phys. Rev. B 65 (2002) 020403(R) [cond-mat/0106525].

[45] J.A. Gracey, Phys. Rev. B 66 (2002) 134402; erratum Phys. Rev. B 70 (2004) 059903. 